\title{
Resource Allocation in Green Dense Cellu
Networks: Complexity and Algorithms
}

\author{
Zoubeir Mlika, Elmahdi Driouch and Wessam Ajib
}

\begin{abstract}
This paper studies the problem of user association, scheduling and channel allocation in dense cellular networks with energy harvesting base stations (EBSs). In this problem, the EBSs are powered solely by renewable energy and each user has a request for downloading data of certain size with a deadline constraint. The objective is to maximize the number of associated and scheduled users while allocating the available channels to the users and respecting the energy and deadline constraints. First, the computational complexity of this problem is characterized by studying its $\mathcal{N} \mathcal{P}$-hardness in different cases. Next, efficient algorithms are proposed in each case. The case of a single channel and a single EBS is solved using two polynomial-time optimal algorithms-one for arbitrary deadlines and a less-complex one for common deadlines. The case of a single channel and multiple EBSs is solved by proposing an efficient constant-factor approximation algorithm. The case of multiple channels is efficiently solved using a heuristic algorithm. Finally, our theoretical analysis is supplemented by simulation results to illustrate the performance of the proposed algorithms.
\end{abstract}

\section{Index Terms}

User association, scheduling, channel allocation, energy harvesting, approximation algorithms, $\mathcal{N} \mathcal{P}$-hardness.

\section{INTRODUCTION}

High spectral efficiency and ultra-low latency are key requirements of 5th generation (5G) cellular networks [1,2]. Initial 5G deployments will focus on enhanced mobile broadband (eMBB) applications with the spectral efficiency being one of the most important key performance indicators [3]. Dense cellular networks (DCNs), where base stations (BSs) are densely deployed in a small geographic area, are considered as an ideal solution to reach such high spectral efficiency. In DCNs, frequency channels are generally allocated based on two approaches: a full frequency reuse and a fractional frequency reuse. In the full reuse approach, all BSs operate on the same channel, which may result in spectral efficiency improvements if the interference is carefully managed [4,5]. Additionally, the BSs consume an important amount of energy [6]. Consequently, an efficient resource allocation for interference management and 
reduced energy consumption are of extreme importance in DCNs. In this paper, resource allocation in DCNs refers to channel allocation, user association, and scheduling, which are three coupled problems that are very hard to solve jointly. Further, energy harvesting and self-powered BSs are deployed in DCNs in order to maximize the use of green energy.

In this paper, we focus on the Resource Allocation problem with Energy and Deadline constraints (we name it $R A E D$ ). In $R A E D$, each user requests to download some data of a given size before a hard deadline. The objective of RAED is to associate and schedule as many users as possible while allocating the available channels to the users subject to the constraints imposed by the request deadlines and the EBSs available energy levels. Due to uncertain and limited levels of the harvested energy and to the coupled nature of this problem, it is very challenging to solve $R A E D$ in DCNs. To the best of our knowledge, previous research literature did not deal with such problem under the same objective and constraints as considered in this paper.

\section{A. Related Work}

The following summarizes the most important work related to our research. In [5] the authors propose a scalable resource allocation approach to solve the channel allocation and user association problem in heterogeneous networks (HetNets) with the objective of minimizing the average packet delay. Their approach iteratively solves a convex optimization problem and an hyper-graph coloring problem. Similar problems to [5] are studied in [7, 8]. In [9], the authors study the problem of channel allocation and power control in non-orthogonal multiple access networks. They use a matching game to design a two-sided exchange-stable algorithm to solve the channel allocation problem. Also, they use sequential convex programming to solve the problem of power control. The authors of [10] study the user association problem where they assume two scenarios: full channel reuse and fractional (orthogonal) channel reuse. They formulate a network utility maximization problem and they use stochastic geometry to obtain the analytical user association bias factors and the channel partition ratios. The works in [5, 7-10] solve the channel allocation or the user association problem without considering energy harvesting BSs nor the scheduling problem. In [11], the authors study multicast scheduling in cellular networks under deadline constraints. Packet scheduling with common deadline is investigated in [12] with the objective of energy minimization. Both [11] and [12] deal exclusively with scheduling without any reference to user association. In [13], channel allocation and scheduling is considered in device-to-device (D2D)enabled DCNs where cellular users have common delay requirements. In [14], the authors consider the problem of real-time packet scheduling in long term evolution advanced (LTE-A) networks. The proposed scheduling algorithm is based on the almost blank subframe (ABS) method to manage the 
interference. In [15], the authors study the scheduling problem in LTE networks based also on $\mathrm{ABS}^{3}$ method. They propose a semi-distributed algorithm to achieve low overhead. In [16], a problem similar to RAED but without channel allocation nor EBSs is considered. The authors show that considering different arrival times of users requests renders the problem $\mathcal{N} \mathcal{P}$-hard even for a single BS network. Next, they develop a constant-factor approximation algorithm to solve the problem. Other related work include packet or job scheduling in the context of scheduling theory [17, 18]. For example, [17] study the problem of real-time job scheduling in an energy harvesting system. All mentioned previous works assume different models and objectives from the model presented in this paper.

Note that most previous research works do not provide theoretical algorithmic analysis of the resource allocation problem in DCNs, e.g., neither $\mathcal{N} \mathcal{P}$-hardness nor approximation algorithms were proposed. In this research, we fill this gap (i) by analyzing the computational complexity of $R A E D$ in different practical cases and (ii) by proposing efficient algorithms with worst-case performance guarantees.

\section{B. Contributions}

This work studies $R A E D$ and its computational complexity in different cases; depending on the number of channels and/or the number of EBSs. The main contributions of this work are summarized in the following list.

- We model $R A E D$ as an integer linear program (ILP) and characterize its computational complexity by studying its $\mathcal{N} \mathcal{P}$-hardness considering the following four cases: (i) the single channel and single EBS (SCSB) case, (ii) the single channel and multiple EBSs (SCMB) case, (iii) the multiple channels and single EBS (MCSB) case, and (iv) the multiple channels and multiple EBSs (MCMB) case.

- For the case of SCSB, we propose

- an optimal polynomial-time (polytime) algorithm for arbitrary deadlines; and

- a less complex optimal polytime algorithm for common deadlines.

- For the case of SCMB, we propose an efficient constant-factor approximation algorithm.

- For the case of MCSB and MCMB, we propose an efficient heuristic algorithm.

- Finally, we show that a preemptive scheduling solution to RAED can be modified, in polytime, to obtain a non-preemptive one.

\section{Organization}

The paper is organized as follows. Section III presents the system model and introduces RAED. Section III formulates RAED and characterizes its computational complexity. Section IV studies 
$R A E D$ in the case of single channel and proposes optimal and approximation algorithms to solve it. Section $\mathrm{V}$ studies $R A E D$ in the case of multiple channels, proposes heuristic algorithms and discusses the non-preemptive scheduling scenario. Section $\mathrm{VI}$ presents the simulations results that illustrate the performance of the proposed algorithms. Finally, Section VII draws some conclusions.

\section{System Model}

We consider a dense cellular network (DCN) composed of $B$ single antenna energy harvesting base stations (EBSs) denoted by the set $\mathcal{B}=\{1,2, \ldots, B\}$. The total bandwidth is divided into a set of $C$ orthogonal channels $\mathcal{C}=\{1,2, \ldots, C\}$ that can be used by the EBSs for downlink transmission. The transmit power of EBS $b$ using any channel is fixed to $P_{b}$ similarly to [5]. Note that the single antenna model is worth studying for the following reasons: (i) it helps to understand the intrinsic difficulty of the problem in the multiple antenna model, (ii) it helps to characterize the structure of the solutions for the multiple antenna model, and (iii) it serves as guideline for the multiple antenna model, i.e. the proposed algorithms for the single antenna model will serve as benchmarks for the algorithms developed in the multiple antenna model.

There are $U$ users in the DCN denoted by the set $\mathcal{U}=\{1,2, \ldots, U\}$. Time is divided into frames where each frame is composed of $T$ slots of duration $\tau$ seconds each. The optimization process to solve the resource allocation problem is performed at the start of each frame. Let $\mathcal{T}=\{1,2, \ldots, T\}$ be the current frame. Every user $u \in \mathcal{U}$ has a data request $\left(s_{u}, d_{u}\right)$ where $s_{u}$ is its size in bits and $d_{u} \in \mathcal{T}$ is its deadline. The EBSs are self-powered thanks to their energy harvesting capabilities. At each time slot $t \in \mathcal{T}$, the amount of harvested energy of EBS $b$ is denoted by $E_{b, t}$ that is stored in EBS's battery assumed to have a large capacity [19]. In the rest of the paper, we normalize $E_{b, t}$ as $A_{b, t}:-E_{b, t} / P_{b}$. Here, $A_{b, t}$ represents the minimum number of slots that can be used by EBS $b$ to transmit with power $P_{b}$ from slot $t$ (at least $A_{b, t}$ slots are available for scheduling starting from $t$ ). By this normalization, energy and slots are treated equivalently in this paper, i.e. when we say energy is available at $t$, it implies that there is at least one slot that can be used from time $s \leqslant t$. Note that, energy arrival $E_{b, t}$ (and hence the number of slots $A_{b, t}$ ) can be any arbitrary non-negative value and it is not assumed to follow any particular distribution, i.e. we consider the general non-stochastic case.

When user $u$ is associated to EBS $b$ using channel $c$, the received signal to interference-plus-noise ratio (SINR) is given by [8]

$$
S I N R_{u, b}^{c}=\frac{P_{b} h_{u, b}^{c}}{\sigma^{2}+\sum_{b^{\prime} \in \mathcal{B} \backslash\{b\}} P_{b^{\prime}} h_{u, b^{\prime}}^{c}},
$$




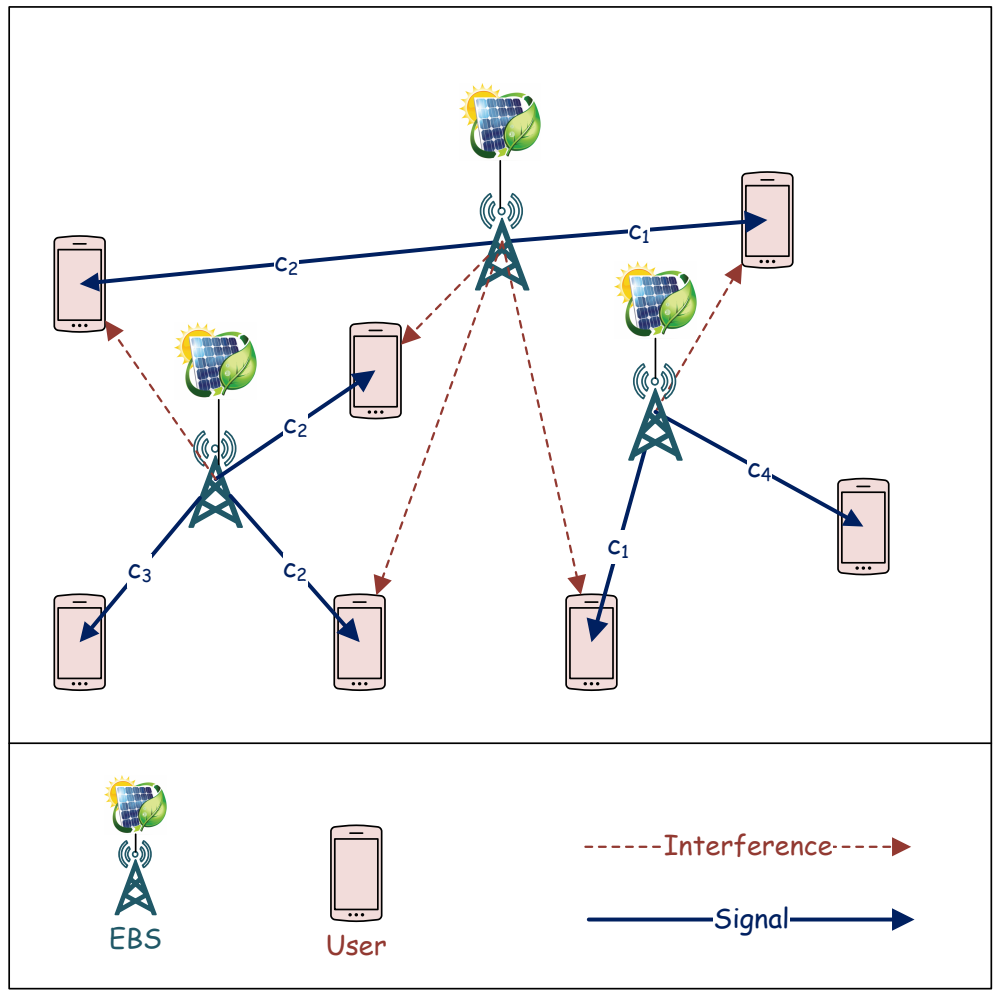

Fig. 1: An example of the system model.

where $\sigma^{2}$ is the power of the additive white Gaussian noise (AWGN) and $h_{u, b}^{c}$ is the channel power gain between user $u$ and EBS $b$ using channel $c$, which takes into account large-scale pathloss propagation effect on a slow timescale [8]. From (1), the achievable data rate, $R_{u, b}^{c}$ (in bps/Hz) of user $u$ when associated to EBS $b$ using channel $c$ can be calculated as

$$
R_{u, b}^{c}=\log _{2}\left(1+S I N R_{u, b}^{c}\right)
$$

User $u$ fulfills its request, when associated to EBS $b$ using channel $c$, if it is allocated $\nu_{u, b}^{c}$ slots, where $\nu_{u, b}^{c}$ is defined as

$$
\nu_{u, b}^{c}:-\left\lceil\frac{s_{u} C}{\tau W R_{u, b}^{c}}\right\rceil,
$$

where $W$ is the total bandwidth in Hz. Hence, in order for user $u$ to download its $s_{u}$ bits, it needs to be associated to EBS $b$ using channel $c$ and scheduled for $\nu_{u, b}^{c}$ slots before its deadline $d_{u}$ and whenever energy is available at EBS $b$. In the rest of the paper, when there is only one EBS (or one channel) in the network, we drop the subscript $b$ (or the superscript $c$ ). An example of the system model is given in Fig. 1, where there are seven users, three EBSs and four frequency channels $\left\{c_{1}, c_{2}, c_{3}, c_{4}\right\}$. There is 
an EBS that schedules two users on channel $c_{2}$ at different time slots.

The objective of this work is to maximize the number of associated and scheduled users in the current frame $\mathcal{T}$ while allocating the channels to the users and satisfying the users demands and respecting the deadlines and energy constraints. RAED is solved in the perfectly-predictable energy arrival model where future energy arrivals are perfectly predicted [20]. Other inputs to RAED are assumed to be known at the beginning of the frame. The non-stochastic online scenario where all inputs are assumed to be unknown is left for future work. Note that the proposed offline algorithms can help us to develop efficient online competitive algorithms in future works. Throughout the rest of the paper, the term served users is used instead of associated and scheduled users.

In the next section, we formulate the problem as an integer linear program and we analyze its computational complexity in different cases.

\section{PRoblem Formulation AND COMPleXity ANALysis}

\section{A. Problem Formulation}

In this subsection, $R A E D$ is formulated as an integer linear program (ILP) to help solving it optimally and efficiently using branch-and-bound algorithm, since brute-force-based approaches are generally inefficient especially when $U, B, C$, or $T$ are large.

To formulate $R A E D$ as an ILP, we introduce the following decision binary variable.

$$
x_{u, b, t}^{c}:- \begin{cases}1, & \text { if } u \text { is associated to } b \text { using channel } c \text { at slot } t, \\ 0, & \text { otherwise. }\end{cases}
$$

Also, let $z_{b, t}$ be the decision integer variable that represents the amount of accumulated number of slots for $\operatorname{EBS} b$ at slot $t$.

The constraints of $R A E D$ can be formulated as follows.

- User $u$ cannot use more than one channel when associated to EBS $b$

$$
x_{u, b, t}^{c}+x_{u, b, t^{\prime}}^{c^{\prime}} \leqslant 1, \quad \forall c \neq c^{\prime},\left(u, b, t, t^{\prime}, c, c^{\prime}\right) \in \mathcal{U} \times \mathcal{B} \times \mathcal{T}^{2} \times \mathcal{C}^{2}
$$

- The users that are associated to EBS $b$ using channel $c$ should not interfere at slot $t$. Two users associated to EBS $b$ using channel $c$ are said to interfere if they are scheduled at the same slot. These constraints are expressed as

$$
\sum_{u \in \mathcal{U}} x_{u, b, t}^{c} \leqslant 1, \quad \forall(b, c, t) \in \mathcal{B} \times \mathcal{C} \times \mathcal{T} .
$$


- User $u$ is associated to only one EBS using channel $c$. We can express these constraints as

$$
x_{u, b, t}^{c}+x_{u, b^{\prime}, t^{\prime}}^{c^{\prime}} \leqslant 1, \quad \forall b \neq b^{\prime},\left(u, b, b^{\prime}, t, t^{\prime}, c, c^{\prime}\right) \in \mathcal{U} \times \mathcal{B}^{2} \times \mathcal{T}^{2} \times \mathcal{C}^{2} .
$$

- The constraints about the update of the number of slots are formulated as

$$
z_{b, t+1}=z_{b, t}+A_{b, t+1}-\sum_{u \in \mathcal{U}} \sum_{c \in \mathcal{C}} x_{u, b, t}^{c}, \quad \forall(b, t) \in \mathcal{B} \times\{1, \ldots, T-1\}
$$

and the initial conditions are

$$
z_{b, 1}=A_{b, 1}, \quad \forall b \in \mathcal{B}
$$

- Each user $u$ is associated to EBS $b$ using channel $c$ only when a positive amount of energy is available at $t$. Hence:

$$
x_{u, b, t}^{c} \leqslant z_{b, t}, \quad \forall(u, b, c, t) \in \mathcal{U} \times \mathcal{B} \times \mathcal{C} \times \mathcal{T},
$$

- Each user $u$ requires $\nu_{u, b}^{c}$ slots when associated to EBS $b$ using channel $c$. These constraints can be written as

$$
x_{u, b, t}^{c} \sum_{s \in \mathcal{T}} x_{u, b, s}^{c}=\nu_{u, b}^{c} x_{u, b, t}^{c}, \quad \forall(u, b, c, t) \in \mathcal{U} \times \mathcal{B} \times \mathcal{C} \times \mathcal{T} .
$$

Note that we multiply both sides of (4) by $x_{u, b, t}^{c}$ because these constraints are only active when user $u$ is associated to EBS $b$ using channel $c$ at slot $t$. Constraints (4) are non-linear. These kind of constraints are known as indicator constraints (ICs), which use binary variables to control whether some linear constraints are active or not. In (4), the binary variable $x_{u, b, t}^{c}$ is used to control whether user $u$ satisfies its required slots $\nu_{u, b}^{c}$ or not. ICs can be easily modeled in modern solvers such as CPLEX [21]. Nonetheless, ICs can be theoretically linearized using the bigM method [22]. Therefore, Constraints (P1h) can be rewritten as

$$
\begin{aligned}
& \sum_{s \in \mathcal{T}} x_{u, b, s}^{c} \geqslant \nu_{u, b}^{c} x_{u, b, t}^{c}, \quad \forall(u, b, c, t) \in \mathcal{U} \times \mathcal{B} \times \mathcal{C} \times \mathcal{T} . \\
& \sum_{s \in \mathcal{T}} x_{u, b, s}^{c} \leqslant \nu_{u, b}^{c} x_{u, b, t}^{c}+M\left(1-x_{u, b, t}^{c}\right), \quad \forall(u, b, c, t) \in \mathcal{U} \times \mathcal{B} \times \mathcal{C} \times \mathcal{T},
\end{aligned}
$$

where $M$ is a large positive number.

We can see from ( $(\mathrm{P1i})$, that if $x_{u, b, t}^{c}=1$, then $\sum_{s \in \mathcal{T}} x_{u, b, s}^{c}=\nu_{u, b}^{c}, \forall(u, b, c, t) \in \mathcal{U} \times \mathcal{B} \times \mathcal{C} \times \mathcal{T}$. Also, if $x_{u, b, t}^{c}=0$, then $0 \leqslant \sum_{s \in \mathcal{T}} x_{u, b, s}^{c} \leqslant M, \forall(u, b, c, t) \in \mathcal{U} \times \mathcal{B} \times \mathcal{C} \times \mathcal{T}$, which is obviously 
true since $M$ is chosen large enough. It is clear that choosing $M=T$ is sufficient.

- The constraints that guarantee the deadline of the users can be expressed as

$$
d_{u} \geqslant t x_{u, b, t}^{c}, \quad \forall(u, b, c, t) \in \mathcal{U} \times \mathcal{B} \times \mathcal{C} \times \mathcal{T}
$$

The objective of $R A E D$ is to maximize the number of served users. Hence, the objective function can be written as

$$
\sum_{u \in \mathcal{U}} \sum_{b \in \mathcal{B}} \sum_{c \in \mathcal{C}} \sum_{t \in \mathcal{T}} x_{u, b, t}^{c} / \nu_{u, b}^{c} .
$$

With that said, we can formulate RAED as the following ILP.

$$
\begin{array}{ll}
\underset{\left[x_{u, b, t}^{c},\left[z_{b, t}\right]\right.}{\operatorname{maximize}} & \sum_{u \in \mathcal{U}} \sum_{b \in \mathcal{B}} \sum_{c \in \mathcal{C}} \sum_{t \in \mathcal{T}} x_{u, b, t}^{c} / \nu_{u, b}^{c} \\
\text { subject to } & x_{u, b, t}^{c} \in\{0,1\}, z_{b, t} \geqslant 0, \quad \forall(u, b, c, t) \in \mathcal{U} \times \mathcal{B} \times \mathcal{C} \times \mathcal{T}, \\
& \text { (P1c) }- \text { P1k. }
\end{array}
$$

Using ILP-based solvers, we can optimally solve (not necessarily in polynomial-time) RAED by solving ( $(\mathrm{P} 1)$. In the next subsection, we analyze the computational complexity of $R A E D$ in different cases. We refer to $R A E D$ in case $X$ as $R A E D-X$. The proposed algorithm to solve $R A E D-X$ is denoted as ALG-X and the optimal algorithm (obtained by solving $\overline{\mathrm{P} 1}$ ) is denoted as OPT-X.

\section{B. Complexity Analysis}

In order to characterize the computational complexity of $R A E D$, we consider four cases: (i) single channel and single EBS (SCSB), (ii) single channel and multiple EBSs (SCMB), (iii) multiple channels and single EBS (MCSB), and (iv) multiple channels, multiple EBSs (MCMB). We summarize the results of our analysis in table $\mathbb{I}$ where $\mathcal{P}$ and $\mathcal{N} \mathcal{P}$ denotes the polytime and the nondeterministic polytime complexity classes, respectively. Table I presents also the proposed algorithms along with their worst-case running-time complexities. We define $L:-\min \{U, B\}$.

The first case of RAED-SCSB is considered in section IV where it is shown that it belongs to the class $\mathcal{P}$, i.e. the polynomial-time (polytime) complexity class.

The second case of RAED-SCMB is analyzed in the following.

Lemma 1. RAED-SCMB is $\mathcal{N} \mathcal{P}$-hard. 
TABLE I: Complexity Classification

\begin{tabular}{lllll}
\hline & \multicolumn{4}{c}{ Cases } \\
\cline { 2 - 5 } & RAED-SCSB & RAED-SCMB & RAED-MCSB & RAED-MCMB \\
\hline Class & $\mathcal{P}$ & NP-hard & NP-hard & NP-hard \\
\hline Alg. & 1) ALG-SCSB & ALG-SCMB & ALG-MCSB & ALG-MCMB \\
& 2) ALG-SCSB & & \\
\hline Type & 1) Optimal & Approximation & Heuristic & Heuristic \\
& 2) Optimal & & & $O\left(B L\left(U^{2} T+U C T^{2}\right)\right)$ \\
\hline Time & 1) $O\left(U T^{2}+T U^{2}\right)$ & $O\left(B L\left(U T^{2}+T U^{2}\right)\right)$ & $O\left(U^{2} T+U C T^{2}\right)$ & \\
\hline
\end{tabular}

Proof: We show that a special case of $R A E D-S C M B$ is $\mathcal{N P}$-hard. Precisely, when all deadlines are identical and energy is always available, $R A E D-S C M B$ is still $\mathcal{N} \mathcal{P}$-hard. An instance of GAP is given by a set of items and a set of bins, where a weight $w_{u, b}$ and a profit $p_{u, b}$ are given for each item $u$ and bin $b$. Further each bin $b$ has capacity $W_{b}$. The objective of GAP is to maximize the profit of the items packed into the bins while respecting the capacity of the used bins. It is known that the special case of $G A P$ where $p_{u, b}=1$ is also $\mathcal{N} \mathcal{P}$-hard [23]. GAP is reduced, in polytime, to RAED-SCMB as follows. Given an instance of $G A P$ with $p_{u, b}=1$, the set of users corresponds to the set of items, the set of EBSs corresponds to the set of bins, $\nu_{u, b}$ corresponds to $w_{u, b}$, and the budget of each EBS $b$ is $W_{b}=T$. It is easy to see that this instance of $R A E D-S C M B$ is solved if and only if GAP is solved. Therefore, an algorithm that solves $R A E D-S C M B$ in polytime can be used to solve GAP in polytime, which is not possible unless $\mathcal{P}=\mathcal{N} \mathcal{P}$. This proves that $R A E D$-SCMB must be also $\mathcal{N} \mathcal{P}$-hard and thereby proves the lemma.

The case of RAED-MCSB is analyzed next.

\section{Lemma 2. RAED-MCSB is $\mathcal{N} \mathcal{P}$-hard.}

Proof: We consider RAED-MCSB when $C=2$, the deadlines are common and there is enough energy available at the EBS. Thus, RAED-MCSB is defined with $B=1, A_{1, t}=T, d_{u}=T$ for all $u$, and $C=2$. In this case, we are given one EBS that has enough energy across all slots, a set of users $\{1,2, \ldots, U\}$ and two channels, where each user $u$ requires $\nu_{u}^{c}$ slots using channel $c=1$ or $c=2$. We are trying to maximize the number of scheduled users at the EBS while respecting the common deadline $T$. We reduce in polytime PARTITION [24] to a decision version of RAED-MCSB. In PARTITION we are given a set of positive integers $\mathcal{S}=\left\{a_{1}, \ldots, a_{S}\right\}$ and we are asked to partition it into two disjoint 
sets $\mathcal{S}_{1}$ and $\mathcal{S}_{2}$ such that $\mathcal{S}_{1} \cup \mathcal{S}_{2}=\mathcal{S}$ and $\sum_{i \in \mathcal{S}_{1}} a_{i}=\sum_{i \in \mathcal{S}_{2}} a_{i}$. Given an instance of PARTITION, we construct an instance of a decision version of $R A E D-M C S B$ as follows. Let $T=\left\lfloor\sum_{i \in \mathcal{S}} a_{i} / 2\right\rfloor$ (assume without loss of generality that $\left.T=\sum_{i \in \mathcal{S}} a_{i} / 2\right)$ and $\nu_{u}^{1}=\nu_{u}^{2}=a_{u}$ for all $u$. The decision version of $R A E D-M C S B$ is: Given this instance, can we schedule all users at the EBS?

On the one hand, if we can solve PARTITION, then all elements of $\mathcal{S}_{1}$ (resp. $\mathcal{S}_{2}$ ) can be scheduled at the beginning of the frame using channel 1 (resp. channel 2). Thus, the decision version of $R A E D$ $M C S B$ is solved. On the other hand, if we can schedule all users using the two channels, then the users scheduled using channel 1 (resp. channel 2) can be chosen to represent the elements of $\mathcal{S}_{1}\left(\right.$ resp. $\left.\mathcal{S}_{2}\right)$. It is clear that $\mathcal{S}_{1} \cup \mathcal{S}_{2}=\mathcal{S}$. Also, since we scheduled all users and $T=\sum_{i \in \mathcal{S}} a_{i} / 2$, then the users scheduled using channel 1 (or channel 2) cannot require more nor less than $\sum_{i \in \mathcal{S}} a_{i} / 2$. Therefore, the users scheduled using channel 1 (or channel 2) require exactly $\sum_{i \in \mathcal{S}} a_{i} / 2$. Consequently, $\sum_{i \in \mathcal{S}_{1}} a_{i}=$ $\sum_{i \in \mathcal{S}_{2}} a_{i}=\sum_{i \in \mathcal{S}} a_{i} / 2$. Thus, PARTITION is solved.

We can see that the created instance of the decision version of $R A E D-M C S B$ is done in polytime and hence it is $\mathcal{N} \mathcal{P}$-hard. This proves the lemma.

Remark. RAED-MCSB can be solved in polytime in a very restricted case. Specifically, when $C \geqslant U$ and each user uses a different channel from every other user and requires the minimum number of slots from the allocated channel, thus, all users can be scheduled at the same slots. Therefore, the following channel allocation is optimal in terms of maximizing the number of scheduled users: for each user $u$, find the channel $c_{u}$ such that $\nu_{u}^{c_{u}}=\min _{c \in \mathcal{C}} \nu_{u}^{c}$. Once the channel allocation is obtained, the problem is reduced to RAED-SCSB, which can be solved by applying the proposed algorithm discussed in section $I V$

Finally, the fourth case of $R A E D-M C M B$ can be shown to be $\mathcal{N} \mathcal{P}$-hard based on the previous results of lemma 1 and lemma 2 .

In order to solve $R A E D$ efficiently, we propose polytime, approximation, or heuristic algorithms depending on the case. We start by studying $R A E D$ with single channel (i.e., $C=1$ ), which represents $R A E D-S C S B$ and $R A E D-S C M B$. In these two cases, $R A E D$ involves only user scheduling and association.

\section{RAED IN THE CASE Of Single CHANNEL}

\section{A. RAED-SCSB}

1) Arbitrary Deadlines: This subsection considers RAED-SCSB when the deadlines of the users are arbitrary. Starting by solving $R A E D-S C S B$ is important as it helps characterizing the structure of the solution in the more general cases. 
First, we introduce the following definition.

Definition 1 (A schedule).

A schedule $\Sigma_{E B S}=\left[\sigma_{1}, \sigma_{2}, \ldots, \sigma_{T}\right]$ of the EBS is an allocation of a set of users $\{1,2, \ldots, U\}$ to a set of slots $\{1,2, \ldots, T\}$. Here, if $\sigma_{t}=u$, then user $u$ is scheduled at slot $t$ (we say that slot $t$ is busy), and if $\sigma_{t}=0$, then slot $t$ is idle (not busy). A preemptive schedule is one where the transmission of some users are interrupted and resumed later on. A non-preemptive schedule is one that is not preemptive. See Fig. 2 for an example.

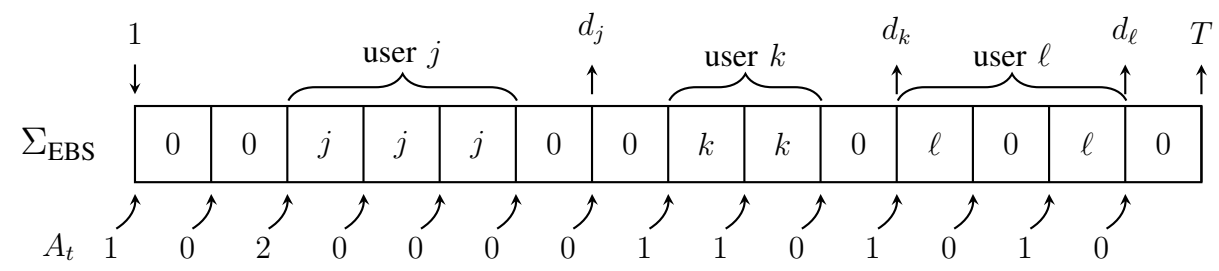

Fig. 2: An example of one EBS and its corresponding preemptive schedule $\Sigma_{\text {EBS }}$.

It is to be noticed that when energy is always available, RAED-SCSB becomes equivalent to maximizing the number of early jobs in a single machine [25], which can be solved optimally using Moore-Hodgson's algorithm [25] that uses a carefully-modified version of the earliest deadline first (EDF) scheduling rule.

To solve RAED-SCSB, we propose a polytime optimal algorithm, called ALG-SCSB 1 , which schedules the maximum number of users while respecting the energy and deadlines constraints. Energy constraints state that users are scheduled only when energy is available and that they should be scheduled for their required number of slots. Deadline constraints state that the scheduled users cannot miss their deadlines. First, we describe ALG-SCSB 1 , then we prove its optimality.

a) Description of $A L G-S C S B_{1}$ : Before going into the details, we start by the following notations and definitions. Lowercase and boldface letters denote vectors whereas uppercase and boldface letters denote matrices. A set and its cardinality are denoted by the same calligraphic and italic letter, respectively. For example, $\mathcal{S}$ denotes a set and $S$ denotes its cardinality. All sets are ordered sets, i.e., the $i$ th element of $\left\{a_{1}, a_{2}, \ldots, a_{S}\right\}$ is $a_{i}$. A matrix $\mathbf{A}$ is sometimes denoted by $\left[a_{i j}\right]$.

Definition 2 (A feasible schedule).

A schedule is called feasible if it is energy-feasible and deadline-feasible. It is energy-feasible if the scheduled users meet the energy constraints and it is deadline-feasible if the scheduled users meet the deadlines constraints. 


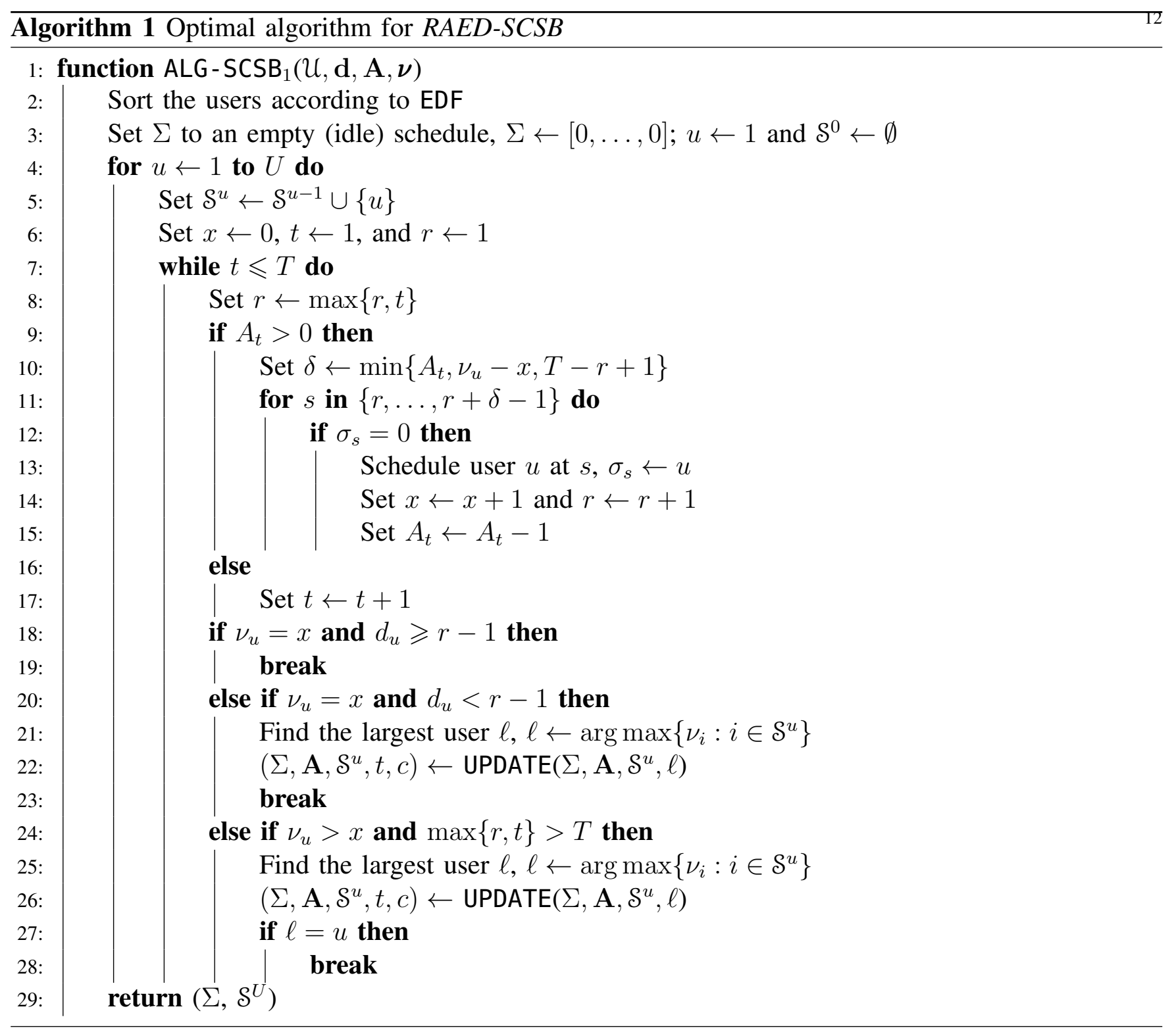

Definition 3 (An $\ell$-optimal schedule).

A schedule $\Sigma^{\ell}=\left[\sigma_{1}^{\ell}, \sigma_{2}^{\ell}, \ldots, \sigma_{T}^{\ell}\right]$ is called $\ell$-optimal, if it is a feasible schedule of the users from $\{1,2, \ldots, \ell\}$ and it schedules the maximum number of users from $\{1,2, \ldots, \ell\}$.

ALG-SCSB 1 is described in the pseudo-code shown in Algorithm 1. It works as follows. First, it sorts the users according to EDF. Then, it starts with an empty (idle) schedule $\Sigma$ (i.e., $\Sigma=[0,0, \ldots, 0]$ ) and it iterates the set of sorted users while checking the energy and deadlines constraints. Let $\mathcal{S}^{u-1}$ be the set of users already scheduled in $\Sigma$ before the start of the $u$ th iteration. At the $u$ th iteration, user

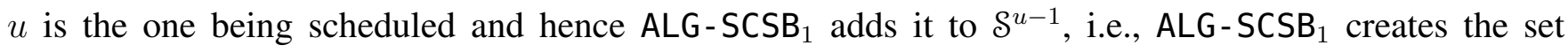
$\mathcal{S}^{u}=\mathcal{S}^{u-1} \cup\{u\}$. Next, ALG-SCSB 1 iterates the slots $t=1,2, \ldots, T$ and schedules $u$ at some slot $s$ 
whenever $A_{t}>0$ for $t \leqslant s$. User $u$ is scheduled at slot $s$ only if $s$ is an idle slot $\left(\sigma_{s}=0\right)$. If $A_{t}={ }_{0}^{13}$, then the next slot is considered. Note that after scheduling user $u$, ALG-SCSB ${ }_{1}$ goes through three if conditions in which it checks the energy and the deadlines constraints. These conditions are given in the following list.

1) User $u$ is allocated $\nu_{u}$ slots and its deadline $d_{u}$ is respected. In this case, the current user is skipped and the algorithm goes to user $u+1$.

2) User $u$ is allocated $\nu_{u}$ slots but its deadline $d_{u}$ is not respected. In this case, ALG-SCSB removes from $\mathcal{S}^{u}$ the largest user $\ell$, updates $\Sigma$ and $\mathbf{A}$, and goes to user $u+1$.

3) User $u$ is not allocated $\nu_{u}$ slots yet (because there is no enough energy) and there is no time left in the frame. In this case, $\mathrm{ALG}-\mathrm{SCSB}_{1}$ removes from $\mathcal{S}^{u}$ the largest user $\ell$, updates $\Sigma$ and $\mathrm{A}$, and

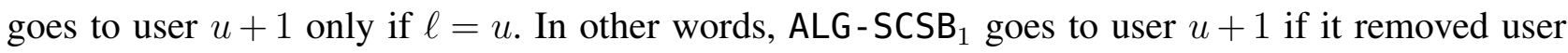
$u$, but continues on scheduling $u$ otherwise.

In the last two conditions, a rescheduling procedure called UPDATE is invoked. It mainly performs three operations: (1) removes $\ell$ from $\mathcal{S}^{u}$, (2) shifts all users scheduled after $\ell$ to the left, and (3) updates the slots (energy) A. Algorithm 2 summarizes the rescheduling procedure. The last two operations of the rescheduling procedure are discussed next. On the one hand, the second operation mainly finds the sets of slots $\mathcal{T}_{1}$ and $\mathcal{T}_{2}$, where $\mathcal{T}_{1}$ represents the slots during which $\ell$ is scheduled whereas $\mathcal{T}_{2}$ is the set of slots during which all users $j \neq \ell$ are scheduled after $\ell$, i.e. $\mathcal{T}_{1}=\left\{t \in \mathcal{T}: \sigma_{t}=\ell\right\}$ and $\mathcal{T}_{2}=\left\{t \in \mathcal{T}: \sigma_{t} \neq 0\right.$ and $\sigma_{t} \neq \ell$ and $\left.t \geqslant \min \mathcal{T}_{1}\right\}$. Next, for each slot $t \in \mathcal{T}_{2}, \sigma_{t}$ is shifted to the left either to a slot of $\mathcal{T}_{1}$ or to a slot of $\mathcal{T}_{2}$ of an already shifted user. Of course, each time a user $u$ is shifted, we must guarantee that it is scheduled at some idle slot where energy is available (its deadline will be respected since it will be shifted to the left). Removing user $\ell$ and shifting all subsequent users update the schedule $\Sigma$ by creating new idle slots in it. On the other hand, the third operation calculates the set of new idle slots $\mathcal{T}_{3}$ in $\Sigma$. For each slot $t \in \mathcal{T}_{3}$, some user in $\Sigma$ was scheduled at $t$ and hence it was allocated one unit of energy at that slot from an earlier slot $s \leqslant t$. Thus, for each slot $t \in \mathcal{T}_{3}$, the third operation calculates the time slot $s$ from which one unit of energy (from $A_{s}$ ) was used at slot $t$ and it updates $A_{s}$ as $A_{s} \leftarrow A_{s}+1$.

After performing these operations, $\mathrm{ALG}-\mathrm{SCSB}_{1}$ returns the tuple $\left(\Sigma, \mathbf{A}, \mathcal{S}^{u}, t, r\right)$. In this tuple, $t$ represents the current time iteration of the while loop of ALG-SCSB,$r$ is the time where the next user will be scheduled, $\Sigma$ is the new schedule and $\mathbf{A}$ is the updated energy.

b) Optimality of ALG-SCSB: Next, we prove that ALG-SCSB ${ }_{1}$ always returns an optimal schedule. First, we show that the returned schedule is feasible and then we prove its optimality. Let $\Sigma^{u}$ be the 


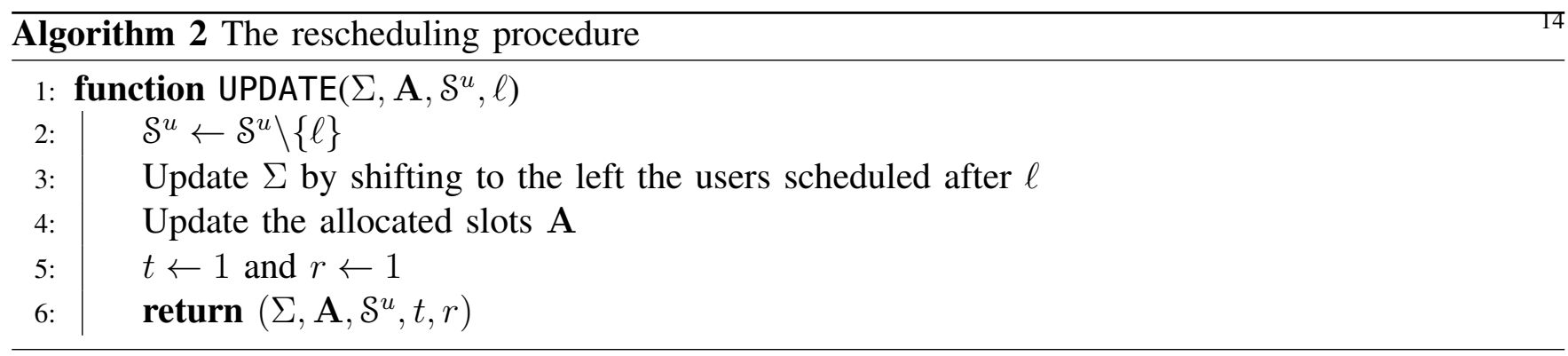

schedule found by ALG-SCSB 1 at the end of iteration $u$, i.e., $\Sigma^{u}$ corresponds to the set $\mathcal{S}^{u}$ of scheduled users. Throughout this part we apply set theory terminology to $\Sigma^{u}$, e.g., $\Sigma^{u-1} \cup\{u\}$ corresponds the set $\mathcal{S}^{u-1} \cup\{u\}$ scheduled according to $\mathrm{ALG}-\mathrm{SCSB}_{1}$.

Lemma 3. The schedule $\Sigma^{u}$ is feasible.

Proof: We prove the lemma by induction. For $u=1$, it is clear that $\Sigma^{1}$ is feasible, since $\Sigma^{1}$ either contains user 1 or is empty and if it contains user 1 then it is clear that it respects the enery and deadline constraints. Assume that $\Sigma^{u-1}$ is feasible. If $\Sigma^{u}=\Sigma^{u-1} \cup\{u\}$ (the rescheduling procedure is not invoked), then we are done. Otherwise (the rescheduling procedure is invoked), let $\ell$ be the user to be removed from $\Sigma^{u-1} \cup\{u\}$, i.e., $\ell$ is the user requiring the largest number of slots, called hereinafter the largest user. We prove that $\Sigma^{u}=\Sigma^{u-1} \cup\{u\} \backslash\{\ell\}$ is feasible. We divide users in $\Sigma^{u}$ into three disjoint sets: (i) users scheduled before $\ell$, (ii) users scheduled after $\ell$, and (iii) user $u$. Notice that:

(i) users scheduled before $\ell$ are left unchanged by the rescheduling procedure (user $j$ in Fig. 3);

(ii) users scheduled after $\ell$ finish earlier due to the shifting operation in the rescheduling procedure. Hence, they respect their deadlines. Also, they respect the energy constraints since $\ell$ is the largest user and $\Sigma^{u-1}$ is energy-feasible (users $i$ and $k$ in Fig. 3); and

(iii) user $u$ respects the energy constraints because $\nu_{u} \leqslant \nu_{\ell}$ and $\Sigma^{u-1}$ is energy-feasible. Also, user $u$ respects its deadline because (1) the last user in $\Sigma^{u}$ finishes before the last user (say $i$ ) in $\Sigma^{u-1}$ due to the shifting operation and to the fact that $\ell$ is the largest user, (2) $d_{u} \geqslant d_{i}$ since users are sorted according to EDF and (3) $\Sigma^{u-1}$ is deadline-feasible. See Fig. 3 .

This proves that $\Sigma^{u}$ is deadline- and energy-feasible and therefore it is feasible. This proves the lemma.

Theorem 1. The schedule $\Sigma^{u}$ is u-optimal for all $u=1,2, \ldots, U$.

Proof: We first prove that for $\ell>u$, there exists an $\ell$-optimal schedule $\Sigma^{\prime \prime}$ that consists of users from $\Sigma^{u}$ and users from $\{u+1, u+2, \ldots, \ell\}$. We proceed by induction. Assume that there exists an 


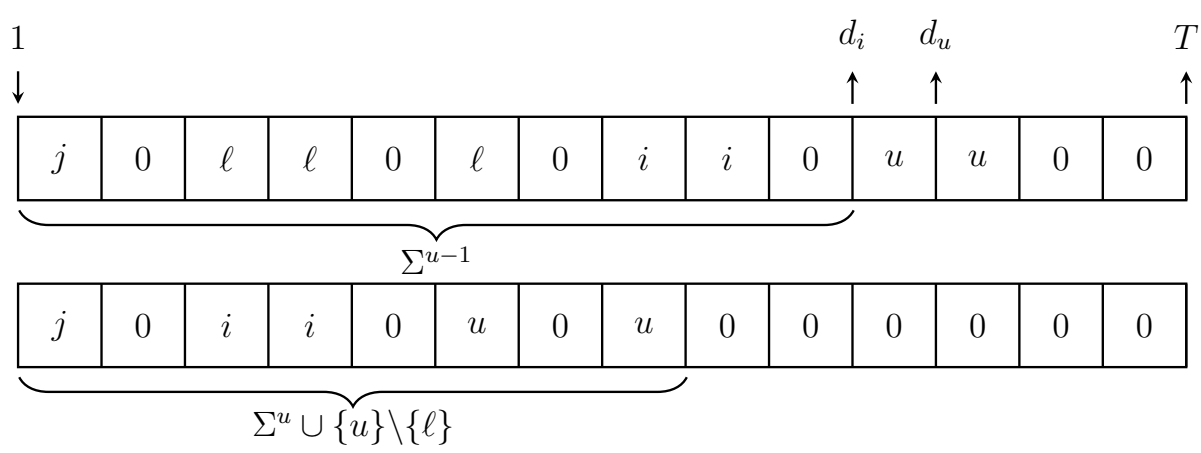

Fig. 3: An illustration of the proof of lemma 3

$\ell$-optimal schedule $\Sigma^{\prime}$ that consists of users from $\Sigma^{u-1}$ and users from $\{u, u+1, \ldots, \ell\}$. We have three cases:

(i) If $\Sigma^{u}=\Sigma^{u-1} \cup\{u\}$. It is clear that an $\ell$-optimal schedule $\Sigma^{\prime \prime}$ exists (i.e., $\Sigma^{\prime \prime}=\Sigma^{\prime}$ ) that consists of users from $\Sigma^{u}$ and users from $\{u+1, u+2, \ldots, \ell\}$.

(ii) If $\Sigma^{u}=\Sigma^{u-1} \cup\{u\} \backslash\{j\}$ where $j \notin \Sigma^{\prime}$. It is also clear that an $\ell$-optimal schedule $\Sigma^{\prime \prime}$ exists (i.e., $\left.\Sigma^{\prime \prime}=\Sigma^{\prime}\right)$ that consists of users from $\Sigma^{u}$ and users from $\{u+1, u+2, \ldots, \ell\}$.

(iii) If $\Sigma^{u}=\Sigma^{u-1} \cup\{u\} \backslash\{j\}$ where $j \in \Sigma^{\prime}$. In this case, to obtain $\Sigma^{\prime \prime}$, we need to modify $\Sigma^{\prime}$. We know that $\Sigma^{u-1} \cup\{u\}$ is not feasible. Hence, there exists $i \in \Sigma^{u-1} \cup\{u\}$ such that $i \notin \Sigma^{\prime}$. Let $\Sigma^{\prime \prime}=\Sigma^{\prime} \cup\{i\} \backslash\{j\}$. It is clear that $\Sigma^{\prime \prime}$ consists of users from $\Sigma^{u}$ and users from $\{u+1, u+2, \ldots, \ell\}$. Also, the number of users in $\Sigma^{\prime}$ is the same as the number of users in $\Sigma^{\prime \prime}$. Hence, it remains to show that $\Sigma^{\prime \prime}$ is feasible. We observe that $\Sigma^{\prime \prime}$ differs from $\Sigma^{\prime}$ only on its intersection with users $\{1,2, \ldots, u\}$, since $i \in \Sigma^{u-1} \cup\{u\}$. We have $\Sigma^{\prime \prime} \cap\{1,2, \ldots, u\} \subseteq \Sigma^{u}$ and, by lemma 3, $\Sigma^{u}$ is feasible. Thus, $\Sigma^{\prime \prime} \cap\{1,2, \ldots, u\}$ is feasible. Based on the previous argument and the fact that ALG - SCSB 1 removes always the largest user (i.e., $\nu_{j} \geqslant \nu_{i}$ ), we conclude that $\Sigma^{\prime \prime}$ is feasible. Finally, we have: for $\ell>u$, there exists an $\ell$-optimal schedule $\Sigma^{\prime \prime}$ that consists of users from schedule $\Sigma^{u}$ and users from $\{u+1, u+2, \ldots, \ell\}$.

We use the previous result and induction to prove that the schedule $\Sigma^{u}$ is $u$-optimal for all $u=$ $1,2, \ldots, U$. For $u=1$, it is clearly true that $\Sigma^{1}$ is 1 -optimal. Suppose that the $\Sigma^{u-1}$ is $u-1$-optimal. From the previous step (for $\ell=u>u-1$ ), we know that there exists a $u$-optimal schedule that consists of users from schedule $\Sigma^{u-1}$ and $\{u\}$. If $\Sigma^{u}=\Sigma^{u-1} \cup\{u\}$, then $\Sigma^{u}$ is $u$-optimal since, by assumption, $\Sigma^{u-1}$ is $u-1$-optimal. Otherwise, the $u$-optimal schedule (i) is a proper subset of users $\Sigma^{u-1} \cup\{u\}$ and (ii) has at least as many users as $\Sigma^{u-1}$ because the latter is $u-1$-optimal. Clearly, $\Sigma^{u} \cup\{u\} \backslash\{j\}$, where $j$ is the largest user, satisfies both conditions (i) and (ii) and hence it is $u$-optimal. Therefore, 
$\Sigma^{u}$ is $u$-optimal. This proves the theorem.

We showed that $\mathrm{ALG}-\mathrm{SCSB}_{1}$ returns an optimal solution to $R A E D-S C S B$. The worst-case complexity of ALG - SCSB 1 is $O\left(U T^{2}+U^{2} T+U \log U\right)=O\left(U T^{2}+U^{2} T\right)$, which is calculated as follows. First of all, it is clear that ALG-SCSB 1 halts in a finite number of steps. The for loop of line 4 halts in $O(U)$ steps. We show that the while loop of line 7 halts in $O(T)$ steps. Let $t$ be an arbitrary iteration of this loop. We can see that if $A_{t}=0$, then the time is incremented. Hence, without loss of generality, assume that $A_{t}>0$ whenever ALG-SCSB 1 enters this loop. With that said, we have three cases: (1) $\nu_{u}=x$ and $d_{u} \geqslant r-1$, (2) $\nu_{u}=x$ and $d_{u}<r-1$, or (3) $\nu_{u}>x$ and $\max \{r, t\}>T$. In the first two cases the while loop halts, since we break it in lines 19 and 23, respectively. The last case is the most expensive one (in terms of time complexity). Whenever $\nu_{u}>x$ and $\max \{r, t\}>T, \mathrm{ALG}-\mathrm{SCSB}_{1}$ calls the rescheduling procedure and goes to the next iteration starting from $t=1$. But, we can see that every time $A_{t}>0$, ALG-SCSB ${ }_{1}$ increases $x$ by at most $\delta$. Thus, at some iteration, $x$ will be greater than or equal to $\nu_{u}$. At this time, ALG-SCSB 1 goes to one of the two previous if conditions and the while loop eventually halts. In the worst-case, $x$ is increased by 1 every time, i.e., $\delta=1$. Hence, the while loop of line 7 halts in $O(T)$ steps. Any iteration of the while loop has a worst-case complexity of $O(U+T)-O(U)$ to find the largest user and $O(T)$ to reschedule the users. Finally, the worst-case complexity of ALG-SCSB 1

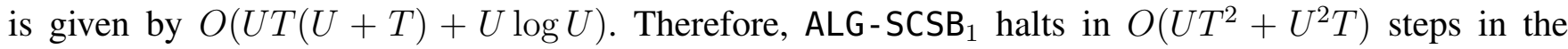
worst-case.

2) Common Deadlines: This subsection solves RAED-SCSB when users have common deadlines, i.e.,

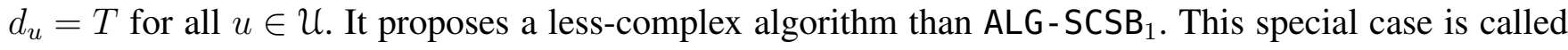
$R A E D-S C S B$ with common deadlines (RAED-SCSB-COMMON). When the energy is always available at the EBS, solving RAED-SCSB-COMMON becomes straightforward. It can be reduced, in polytime, to an unweighted knapsack problem as follows. The users represent the items where the weight of item $u$ is given by the required slots $\nu_{u}$, the EBS represents the knapsack and the common deadline $T$ is the knapsack capacity. Hence, RAED-SCSB-COMMON when the energy constraints are omitted can be solved optimally by sorting the users in increasing order of their required slots and scheduling the users one after the other until $T$ is reached. This procedure, named PACK, is applied at the beginning of the frame (i.e., at $t=1$ ) and accepts as inputs the set of users $\mathcal{U}$, the common deadline $T$, the required number of slots $\boldsymbol{\nu}$, and the time $t$. The worst-case time complexity of PACK is $O(T+U \log U)$, where $O(U \log U)$ steps are required to find the users to be scheduled and $O(T)$ steps are required to create the schedule.

Now, to solve RAED-SCSB-COMMON with energy constraints, we reduce it, in polytime, to an 
unweighted knapsack problem with cumulative capacity; that is, the capacity of the knapsack is not fixed to $T$ but changes from one slot to another depending on the energy arrivals. Therefore, knowing the maximum accumulated capacity, we can apply PACK to obtain an optimal solution to RAED-SCSBCOMMON. Let $\Lambda_{t}$ be the accumulated capacity at time $t$, which is defined as the maximum number of slots that can be used by the EBS from slot $t$. The cumulative capacity procedure that calculates $\Lambda_{t}$ for $t \in \mathcal{T}$ works iteratively as follows. In the first iteration, it calculates $\Gamma(t):-\sum_{i=1}^{t} A_{i}$. If no energy arrives during the period $\{t+1, \ldots, t+\Gamma(t)\}$, then no more than $\Gamma(t)$ slots can be used by the EBS and thus $\Lambda_{t}=\Gamma(t)$. Otherwise, $\Lambda_{t}>\Gamma(t)$. In fact, $\Lambda_{t}=\Gamma(t+\Gamma(t))$. Similarly, in the second iteration, if no energy arrives in the period $\left\{t^{\prime}+1, \ldots, t+\Gamma\left(t^{\prime}\right)\right\}$, then $\Lambda_{t}=\Gamma\left(t^{\prime}\right)$. Otherwise, $\Lambda_{t}=\Gamma\left(t+\Gamma\left(t^{\prime}\right)\right)$ and the process continues this way. Of course the final accumulated capacity cannot exceed the remaining number of slots. Hence, $\Lambda_{t}=\min \left\{\Gamma\left(t^{\prime}\right), T-t+1\right\}$, where $t^{\prime}$ is the iteration such that no energy arrived in the period $\left\{t^{\prime}+1, \ldots, t+\Gamma\left(t^{\prime}\right)\right\}$. This procedure is illustrated in Algorithm 3 and is called BUDGET. After calculating the accumulated capacity $\Lambda_{t}$ at each time $t$, BUDGET finds the maximum capacity

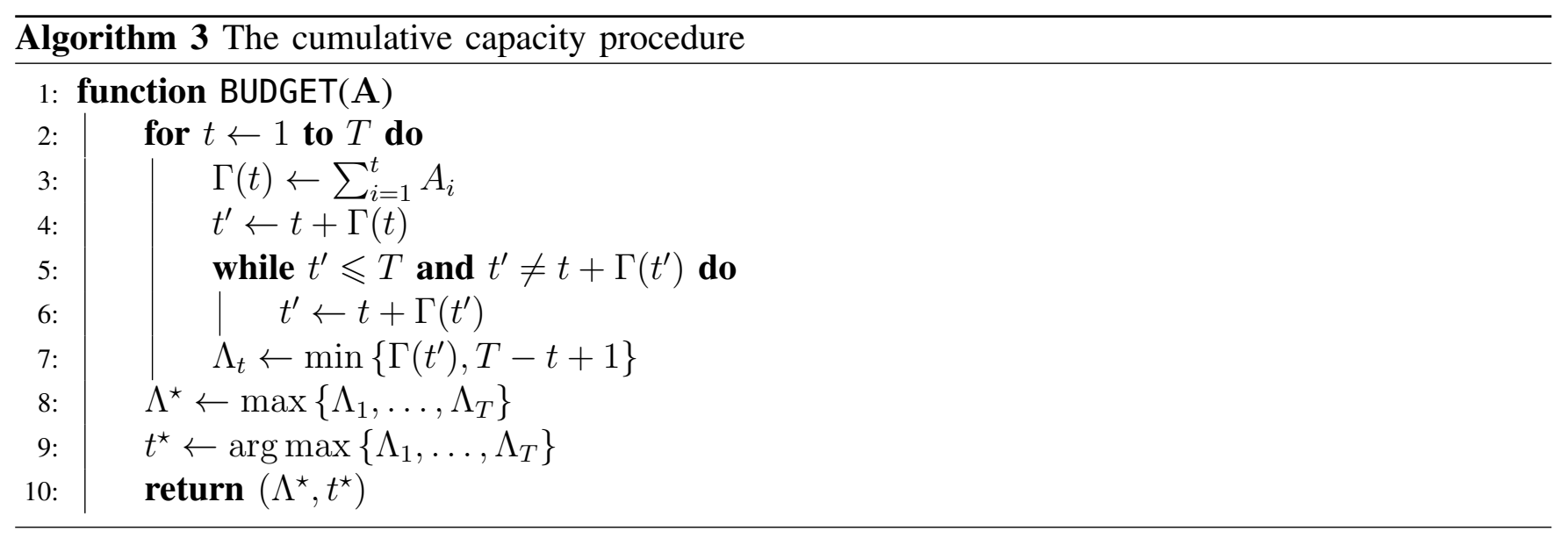

$\Lambda^{\star}=\Lambda_{t^{\star}}=\max \left\{\Lambda_{1}, \ldots, \Lambda_{T}\right\}$ where $t^{\star}=\arg \max \left\{\Lambda_{1}, \ldots, \Lambda_{T}\right\}$. Given $\Lambda^{\star}$ and $t^{\star}$, the algorithm that solves $R A E D-S C S B-C O M M O N$ calls $\operatorname{PACK}\left(\mathcal{U}, \Lambda^{\star}, \boldsymbol{\nu} ; t=t^{\star}\right)$, which is applied at $t=t^{\star}$. Since $\Lambda^{\star}$ represents the maximum possible number of slots that can be used by the EBS, then this leads to an optimal solution to RAED-SCSB-COMMON.

This algorithm, called ALG-SCSB 2 , finds an optimal solution to RAED-SCSB-COMMON in $O\left(T^{2}+\right.$ $T+U \log U)=O\left(T^{2}+U \log U\right)$ steps in the worst-case, where $O\left(T^{2}\right)$ steps are required to calculate the accumulated capacity $\Lambda^{\star}$ and $O(T+U \log U)$ steps are required to schedule the users by calling PACK. We can see that ALG-SCSB 2 is much simple (less complex) than ALG-SCSB 1 for arbitrary deadlines. This simplicity is obtained by exploiting the structure of the problem in which the users have common deadlines. 
In the next section, we study $R A E D$ in the single channel case but with multiple EBSs, i.e., $R A E D$ $S C M B$.

\section{B. RAED-SCMB}

We showed in Section III that $R A E D-S C M B$ is $\mathcal{N} \mathcal{P}$-hard. Hence, we aim to design a polytime approximation algorithm to solve RAED-SCMB. Approximation algorithms [26] are algorithms that find "good enough" solutions very fast, where goodness is measured by the ratio of the value of the algorithm to the value of the optimal (e.g. brute-force) algorithm. In other words, approximation algorithms are polytime algorithms that are guaranteed theoretically to find solutions that are close to the optimal ones.

1) An Approximation Algorithm for RAED-SCMB: To solve RAED-SCMB, we propose ALG-SCMBan iterative algorithm that uses ALG-SCSB 1 as a subroutine. In short, for each iteration, ALG-SCMB finds the EBS that can schedule the maximum number of users. The pseudo-code of ALG-SCMB is given in Algorithm 4. We use the following additional notations. The collection of sets $\mathcal{A}_{\mathcal{B}}$ denotes $\left\{\mathcal{A}_{i}: i \in \mathcal{B}\right\}$, where $\mathcal{A}_{i}$ is a set. Further, given a matrix $\mathbf{A}$, we use $\mathbf{A}_{i}$ to denote its $i$ th row and $\mathbf{A}^{i}$ to denote its $i$ th column.

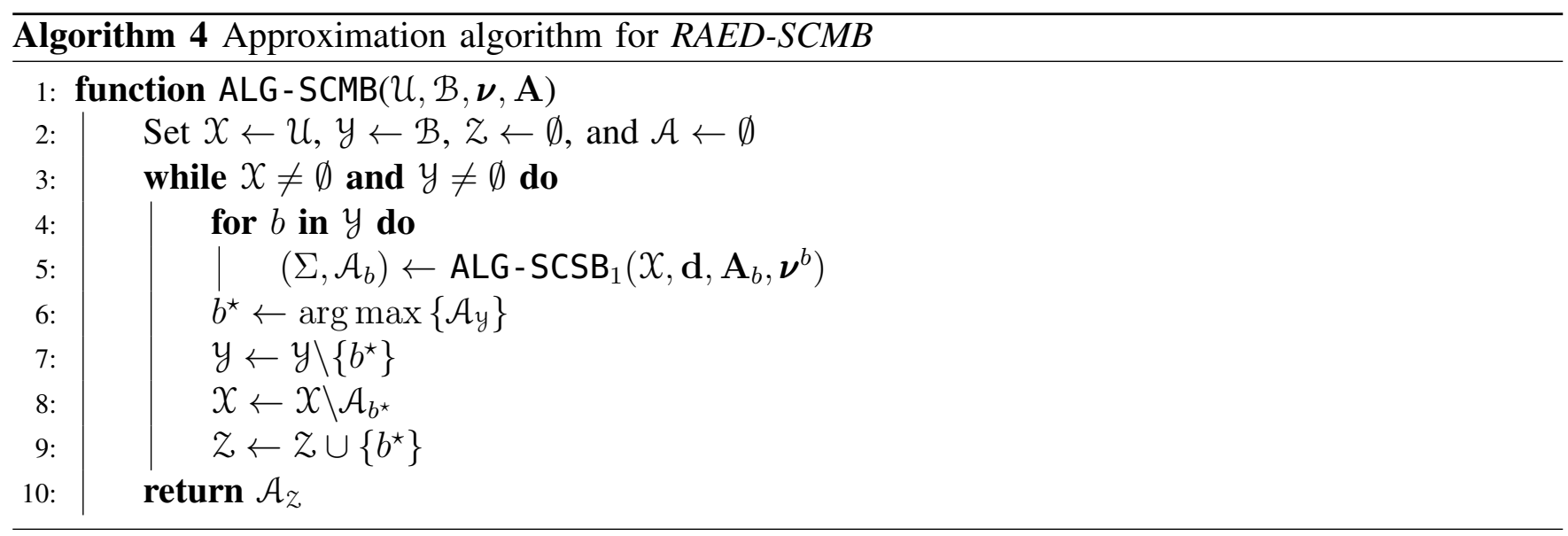

In more details, ALG-SCMB works as follows. Let $\mathcal{X}$ (resp. $\mathcal{Y}$ ) be the set of remaining users (resp. the set of remaining EBSs) after any iteration. Initially, $X=\mathcal{U}$ and $y=\mathcal{B}$. For each iteration, ALG-SCMB goes through $y$ and schedules the users in $X$ according to ALG-SCSB ${ }_{1}$. At the end of this iteration, ALG - SCMB creates a set $\mathcal{A}_{b}$ of scheduled users to every EBS $b$. Next, it selects EBS $b^{\star}$ that schedules the maximum number of users $\mathcal{A}_{b^{\star}}$. Then, it updates the set $X$ by removing the users $\mathcal{A}_{b^{\star}}$ and updates the set $y$ by removing EBS $b^{\star}$. At every iteration, ALG-SCMB updates the set $z$ of used EBSs by adding EBS $b^{\star}$. And, when ALG-SCMB halts, it returns the tuple $\mathcal{A}_{z}$ of served users. 
We can show that ALG-SCMB is a constant-factor approximation algorithm for RAED-SCMB. This means that ALG-SCMB always returns a solution to RAED-SCMB (i) in polytime, and (ii) whose value is bounded below by a constant times the optimal value. The following lemma proves this result.

Lemma 4. ALG-SCMB is a $\frac{1}{2}$-approximation algorithm for RAED-SCMB.

Proof: We prove the lemma by showing that ALG-SCMB (i) halts in polytime, and (ii) the number of served users by ALG-SCMB is at least half the optimal number. The first point follows from the fact that ALG-SCSB 1 is a polytime algorithm and that the while loop in line 3 of Algorithm 4 halts in $L:-\min (U, B)$ steps. Hence, ALG-SCMB halts in $O\left(B L\left(U T^{2}+U^{2} T\right)\right)$ steps in the worst case. To prove the second point, let OPT-SCMB be an optimal algorithm for $S A E D-S C M B$ and let $\mathcal{O}_{b}$ be the set of users served by EBS $b$ in OPT-SCMB but which are not considered by any EBS in ALG-SCMB. Note that we do not have to know the elements of $\mathcal{O}_{b}$ neither the optimal algorithm OPT-SCMB. According to our notations in Algorithm 4, $\mathcal{A}_{b}$ corresponds to the set of users served by EBS $b$ in ALG-SCMB. Define $X_{b}:-\left|\mathcal{A}_{b}\right|$. Finally, let $\mathcal{C}_{b}$ be the set of common users (i.e. users served by EBS $b$ in both OPT-SCMB and ALG-SCMB). The optimal number of served users in OPT-SCMB is $X^{\star}:-\sum_{b=1}^{B}\left|\mathcal{O}_{b}\right|+\left|\mathcal{C}_{b}\right|$ whereas the number of served users in ALG-SCMB is $X:-\sum_{b=1}^{B} X_{b}$. For any EBS $b$, all users in $\mathcal{O}_{b}$ can be considered by ALG-SCMB. Since ALG-SCMB serves the maximum number of remaining users, then EBS $b$ must serve at least $\left|\mathcal{O}_{b}\right|$, i.e., we have $X_{b} \geqslant\left|\mathcal{O}_{b}\right|$, for all EBSs $b=1,2, \ldots, B$. Thus, summing over b, we obtain:

$$
X \geqslant \sum_{b=1}^{B}\left|\mathcal{O}_{b}\right| .
$$

Now, there are two cases: whether or not the total number users served only by OPT-SCMB is greater than or equal to half the optimal number $X^{\star}$.

- If $\sum_{b=1}^{B}\left|\mathcal{O}_{b}\right| \geqslant X^{\star} / 2$. In this case, based on (6), we have $X \geqslant X^{\star} / 2$.

- If $\sum_{b=1}^{B}\left|\mathcal{O}_{b}\right| \leqslant X^{\star} / 2$. In this case, we have $\sum_{b=1}^{B}\left|\mathcal{O}_{b}\right|+\sum_{b=1}^{B}\left|\mathcal{C}_{b}\right| \leqslant X^{\star} / 2+\sum_{b=1}^{B}\left|\mathcal{C}_{b}\right|$ and hence $X^{\star} \leqslant X^{\star} / 2+\sum_{b=1}^{B}\left|\mathcal{C}_{b}\right|$. Alternatively, $X^{\star} / 2 \leqslant \sum_{b=1}^{B}\left|\mathcal{C}_{b}\right|$. Since the users in $\mathcal{C}_{b}$ are served by both ALG-SCMB and OPT-SCMB, then $\left|\mathcal{C}_{b}\right| \leqslant X_{b}$ for all $b$. Thus, $\sum_{b=1}^{B}\left|\mathcal{C}_{b}\right| \leqslant X$. Finally, we obtain $X \geqslant X^{\star} / 2$.

In both cases, we have $X^{\star} \geqslant X \geqslant X^{\star} / 2$. Therefore, ALG-SCMB is a $\frac{1}{2}$-approximation algorithm for $R A E D-S C M B$. This proves the lemma.

Remark. The approximation factor of $1 / 2$ of ALG -SCMB is tight. That is, no better factor can be obtained for ALG-SCMB. This is shown in the following example. Let $U=4, B=2, T=2$, and $A_{11}=2$, 
$A_{12}=0, A_{21}=2, A_{22}=0$. The required slots are given by $\nu_{11}=\nu_{21}=\nu_{22}=\nu_{31}=\nu_{32}=\nu_{41}=\stackrel{20}{1}$ and $\nu_{42}=\nu_{12}=2$. On the one hand, the optimal algorithm associates users 1 and 4 to EBS 1 and schedules them at slots 1 and 2, respectively, and associates users 2 and 3 to EBS 2 and schedules them at slots 1 and 2, respectively. Hence, the optimal algorithm serves 4 users. On the other hand, if ALG-SCMB associates users 2 and 3 to EBS 1 and schedules them at slots 1 and 2, respectively, then it can only associate user 1 or user 4 to EBS 2 and schedules it at either slot 1 or 2. Hence, ALG-SCMB serves 2 users. We can see that $X=X^{\star} / 2$. Therefore, the approximation factor $1 / 2$ of ALG-SCMB is tight.

\section{RAED in the CASE of Multiple Channels}

This section considers $R A E D$ in the case of multiple channels (i.e., RAED-MCSB and RAED-MCMB). The solution in this case is based on a three-steps procedure inspired by the algorithms developed for the single channel case. In the first step, the channel allocation solution is obtained by a greedy approach. In the second step, the user scheduling for a single EBS is derived by modifying ALG-SCSB ${ }_{1}$. Finally, the third step is to find the association solution, which is obtained by iteratively applying the two first steps. In order to detail this three-steps solution, we first consider RAED-MCSB and describe a heuristic algorithm by modifying ALG $-\mathrm{SCSB}_{1}$. Next, we solve RAED-MCMB by proposing an iterative algorithm.

\section{A. RAED-MCSB}

To solve RAED-MCSB, we propose a heuristic algorithm, called ALG-MCSB, that first allocates the channels to the users that require the least number of slots. In other words, user $u$ is allocated the channel $c_{u}$ such that

$$
c_{u}=\underset{c \in \mathcal{C}}{\arg \min } \nu_{u}^{c} .
$$

In the case of a tie, user $u$ is allocated the channel that is least used. Once the channel allocation is obtained, ALG-MCSB schedules the users to the EBS by applying a modified version of ALG-SCSB . $_{\text {. }}$ The schedule $\Sigma$ is now a matrix $\left[\sigma_{t c}\right]$ where $\sigma_{t c}=u$ iff user $u$ is scheduled at slot $t$ using channel $c$. Hence, ALG-MCSB (including the rescheduling procedure) works with the matrix $\left[\sigma_{t c}\right]$ and has to verify that two users cannot be scheduled at the same slot using the same channel.

\section{B. RAED-MCMB}

This subsection solves RAED-MCMB by applying iteratively ALG-MCSB in each EBS. In every iteration, two main steps are preformed: (1) the EBS that maximizes the number of served users is found 
and (2) the served users are removed from the whole set of users. The idea of this iterative algorithm (called ALG-MCMB) is similar to the one of the previously proposed approximation algorithm ALG -SCMB. ALG-MCMB is described in the following pseudo-code where the same notations as in Algorithm 4 are used.

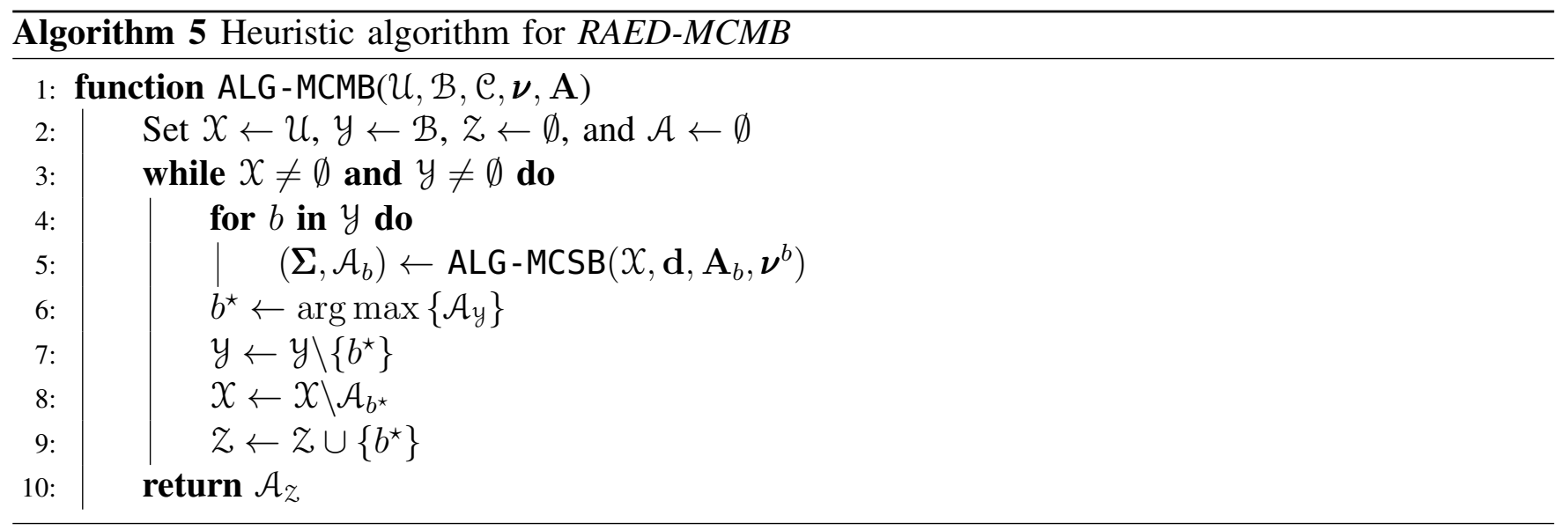

Note that ALG-MCMB calls ALG-MCSB in line 5 where the latter finds the channel allocation according to (7). Based on the analysis of the worst-case complexity of ALG-SCSB ${ }_{1}$, we obtain the worst-case complexity of ALG-MCSB as $O(C+U \log U+U T(U+C T))=O\left(U^{2} T+U C T^{2}\right)$. Therefore, the worst-case complexity of ALG-MCMB is $O\left(B L\left(U^{2} T+U C T^{2}\right)\right)$ where $L:-\min \{U, B\}$.

Of course, since RAED-MCSB is $\mathcal{N P}$-hard, neither ALG-MCSB nor ALG-MCMB is guaranteed to give the optimal solution to RAED-MCSB or RAED-MCMB. Nonetheless, note that the greedy strategy used to allocate the channels can be seen as the best possible locally, i.e., for user $u$, it is optimal to choose the channel that uses fewer number of slots in order to satisfy its request.

In the next subsection, we illustrate how the scheduling solutions obtained in the previous parts of the paper can be modified to be non-preemptive.

\section{Non-Preemptive Scheduling}

In this section, we discuss $R A E D$ with non-preemptive scheduling constraints. In other words, we further constrain the problem by imposing that users' requests should not be interrupted. Without loss of generality, we consider the case of $B=C=1$. We start by the following definition.

Definition 4 (Starting and completion times).

Given a schedule $\Sigma=\left[\sigma_{1}, \sigma_{2}, \ldots, \sigma_{T}\right]$, we define the starting time $S_{u}$ (resp. completion time $C_{u}$ ) of user $u$ as the smallest (resp. the largest) time for which $\sigma_{S_{u}}=u$ (resp. $\sigma_{C_{u}}=u$ ). 
We show that the schedule returned by $A L G-\mathrm{SCSB}_{1}$ can be modified, in polytime, to obtain a new non-

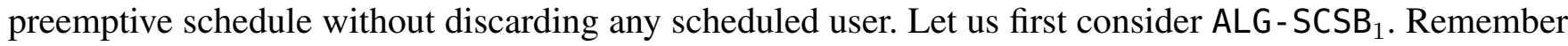
that $\Sigma^{u}$ is the schedule that corresponds to the set of scheduled users $\mathcal{S}^{u}$ given by ALG-SCSB 1 at the end of iteration $u$.

Lemma 5. The schedule $\Sigma^{u}$ can be transformed, in polytime, to a non-preemptive one without discarding any user.

Proof: Assume without loss of generality that an arbitrary user $i$ is scheduled preemptively in $\Sigma^{u}$. Let $S_{i}$ and $C_{i}$ be the starting and completion times of $i$. Since the scheduling of $i$ is preempted, then $C_{i}-S_{i}+1>\nu_{i}$ and $C_{i}-S_{i}-\nu_{i}+1$ are the number of slots that remain empty in $\left\{S_{i}, \ldots, C_{i}\right\}$ due to preemption. First, note that no user other than $i$ is scheduled in the idle slots of $\left\{S_{i}, \ldots, C_{i}\right\}$ because, otherwise, $i$ would have been scheduled instead. Second, $i$ can be scheduled at time $S_{i}^{\prime}=C_{i}-\nu_{i}+1$. Doing so, we guarantee that: (i) $i$ is scheduled non-preemptively since $C_{i}-S_{i}^{\prime}+1=\nu_{i}$, (ii) the deadline constraint of $i$ is always met since $C_{i}$ is kept unchanged, and (iii) the energy constraint is also met since the slots used in $\left\{S_{i}, \ldots, S_{i}^{\prime}\right\}$ can be used in $\left\{S_{i}^{\prime}, \ldots, C_{i}\right\}$. This proves that the schedule $\Sigma^{u}$ can be transformed into a non-preemptive one in polytime without discarding any scheduled user. Hence, the lemma is proved.

Lemma 5 shows that even if a scheduling solution to RAED is preempted, it is possible to change it to a non-preemptive one without decreasing the objective function. This is due primarily to the structure of the proposed algorithms and to the sufficiently large battery capacity.

In the next section, we present simulation results to show the performance of the proposed algorithm.

\section{Simulation Results}

\begin{tabular}{l|l} 
Parameter & Value \\
\hline EBS transmit power & $30 \mathrm{dBm}$ \\
\hline Total bandwidth $W$ & $20 \mathrm{MHz}$ \\
\hline Carrier frequency & $2 \mathrm{GHz}$ \\
\hline Noise power density & $-174 \mathrm{dBm} / \mathrm{Hz}$ \\
\hline Path loss between user $u$ and EBS $b$ & $30.6+36.7 \log _{10}$ dist $_{u, b}$ \\
\hline Energy arrival rate & $\lambda=0.5$ \\
\hline Request data size & $s_{u} \sim$ unif $\{1 K b, 1 M b\}$ for all $u \in \mathcal{U}$ \\
\hline Frame length & $T=10$ \\
\hline Deadlines & $d_{u} \sim \operatorname{unif}\{1, T\}$ for all $u \in \mathcal{U}$ \\
\hline Number of EBSs & $B=10$ \\
\hline
\end{tabular}

TABLE II: Simulation parameters. 
In this section, we present simulation results to illustrate the performance of the proposed algorithms. We consider a geographical area of size $20 \times 20$ square meters where the users and EBSs are randomly and uniformly distributed. The channel gains are based on 3GPP specifications [27] and are modeled similarly to [8]. The energy arrival $E_{b, t}$ is assumed to follow a Poisson distribution with parameter $\lambda$. Unless otherwise specified, simulations use the parameters shown in Table II The optimization problem $(\mathrm{P} 1)$ is modeled in Python using PuLP [28] and solved using the CPLEX solver [21]. The simulations are performed on 1000 realizations and averaged out, where in each realization independent random parameters are generated.

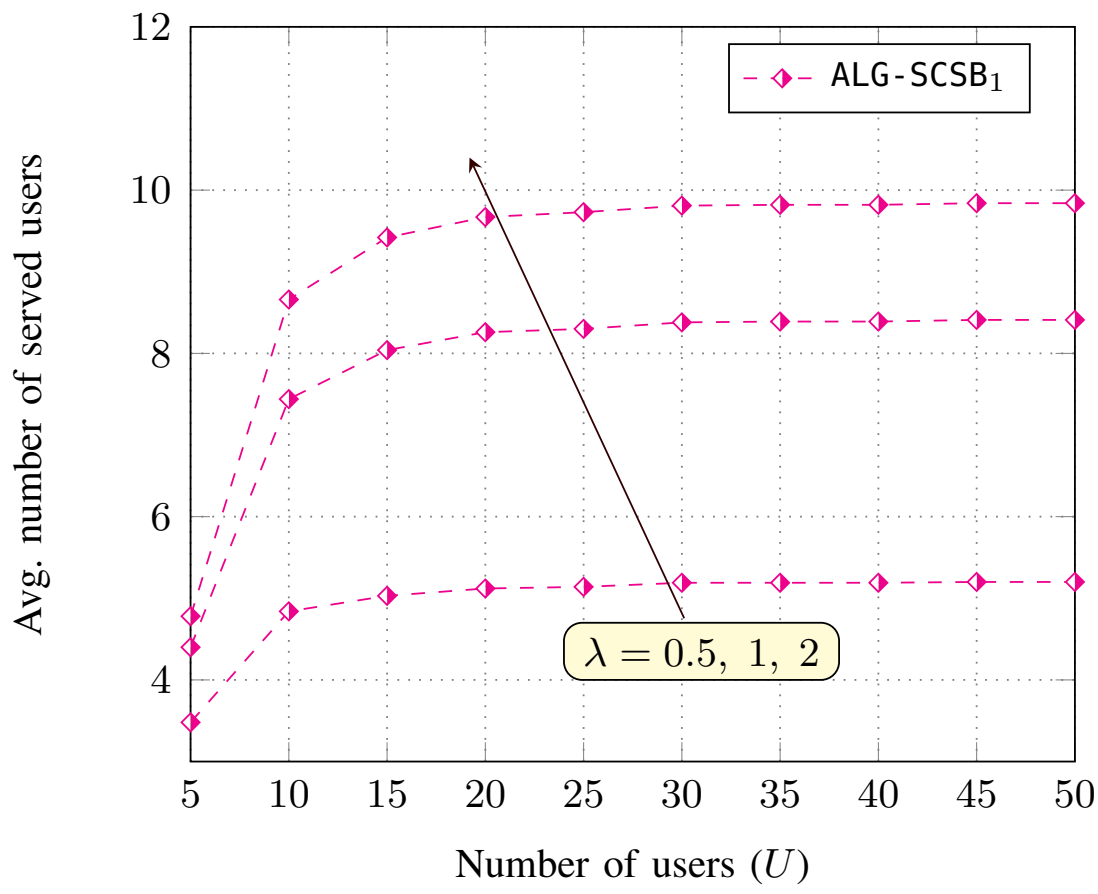

Fig. 4: Average number of served users by ALG-SCSB 1 for different $\lambda$.

Fig. 4 illustrates the performance of $\mathrm{ALG}-\mathrm{SCSB}_{1}$ when varying both $U$ and $\lambda$. As $\lambda$ increases, more users are served. Since the number of slots is fixed to $T=10$, no more than 10 users can be served in the best case (when every user requires one unit of energy). It is also clear that as $\lambda$ increases, the number of served users increases rapidly to reach 10 . For example, for a fixed $U$, the gap in the y-axis from $\lambda=0.5$ to $\lambda=1$ is larger than the gap from $\lambda=1$ to $\lambda=2$. This is because as $\lambda$ increases, the energy will be always available and the problem becomes equivalent to scheduling users in increasing order of required energy.

Fig. 5 shows the performance of ALG-SCSB 1 against $U$ and $T$. It is clear that as $T$ increases, more users can be served. We can see that, e.g., for $T=20$, the number of served users is about 10 , which 


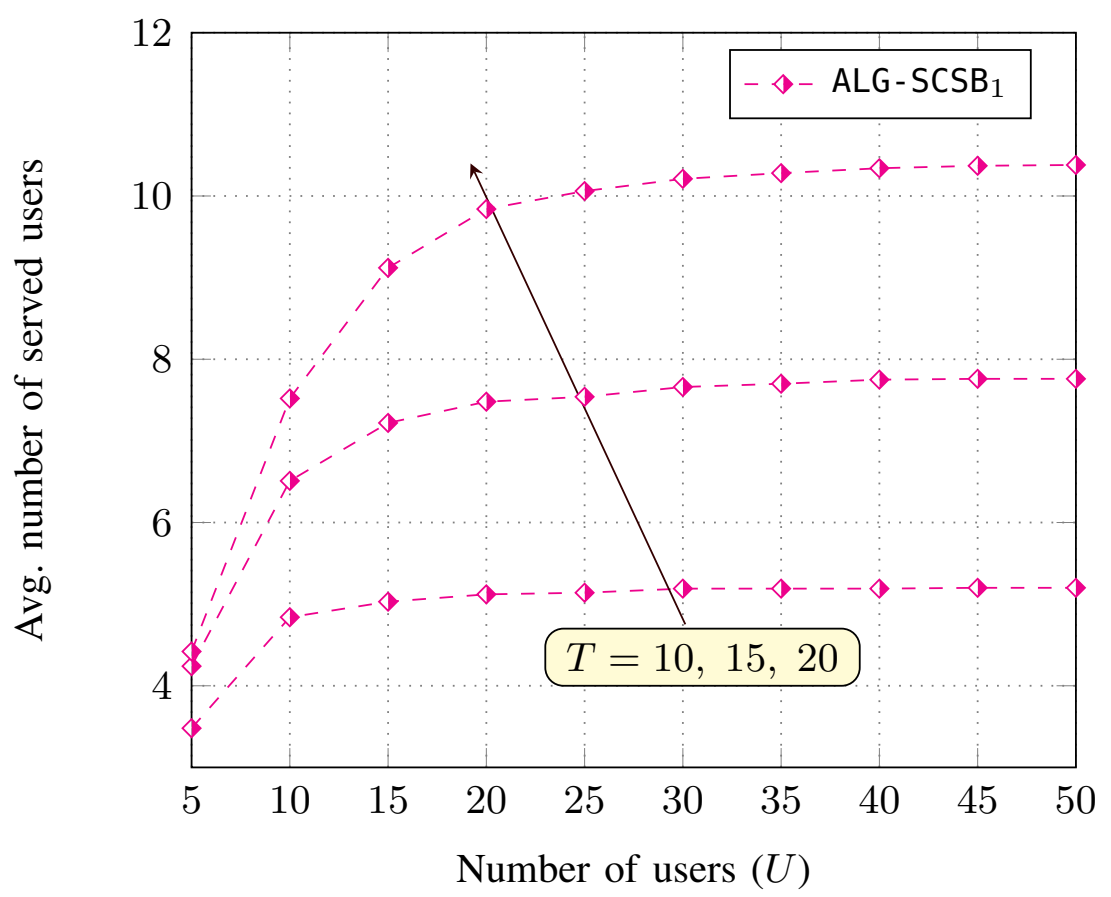

Fig. 5: Average number of served users by ALG-SCSB 1 for different $T$.

indicates that the users require on average 2 units of energy (i.e., $\nu_{u} \approx 2$ ). In fact, as the number of users increases, not all of them can be served even for larger values of $T$. This is due to the required number of slots by each user and also by the energy availability at the EBS.

In Fig. 6, we compare the performance of ALG -SCMB to OPT-SCMB when varying $U$ and $\lambda$. We can see that ALG-SCMB performs close to OPT-SCMB even for large values of $U$. As $\lambda$ increases, energy will be always available and hence the problem becomes easier; though it is still $\mathcal{N} \mathcal{P}$-hard. Consequently, ALG SCMB performs closer to OPT-SCMB for large values of $\lambda$ than for small ones. Notice that the average performance of ALG-SCMB is better than the theoretical guarantee given by the approximation ratio of 0.5 (see lemma 4). This is clear from the figure where ALG-SCMB achieves on average a performance ratio (optimal-to-algorithm) of 0.925 for $U=50$ and $\lambda=0.5$.

Fig. 7 compares ALG - SCMB to OPT - SCMB for different values of $U$ and $T$. Both algorithms are close to each other. When $T$ gets larger and for fixed $U$ and $B$, the gap between both algorithms shrinks. For $T=15$ and despite the small gap between both algorithms, we can see that it increases slowly as $U$ increases. Interestingly, similarly to Fig. 6, ALG-SCMB has a better average performance compared to the theoretical performance guarantee.

Fig. 8 illustrates the performance of ALG -SCMB against OPT-SCMB when varying $U$ and $B$. For small and fixed $B$, ALG-SCMB gets closer to OPT-SCMB as $U$ increases. This is because the maximum possible 


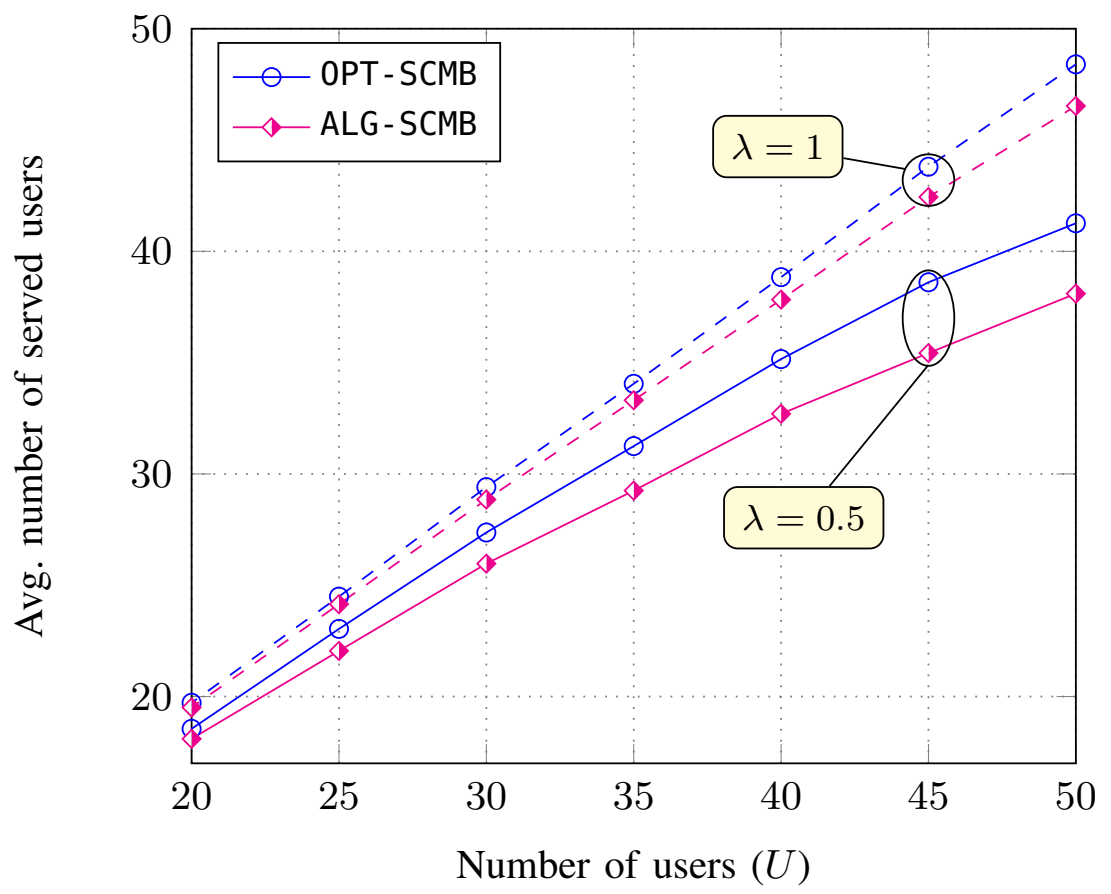

Fig. 6: Average number of served users by ALG-SCMB and OPT-SCMB for different $\lambda$.

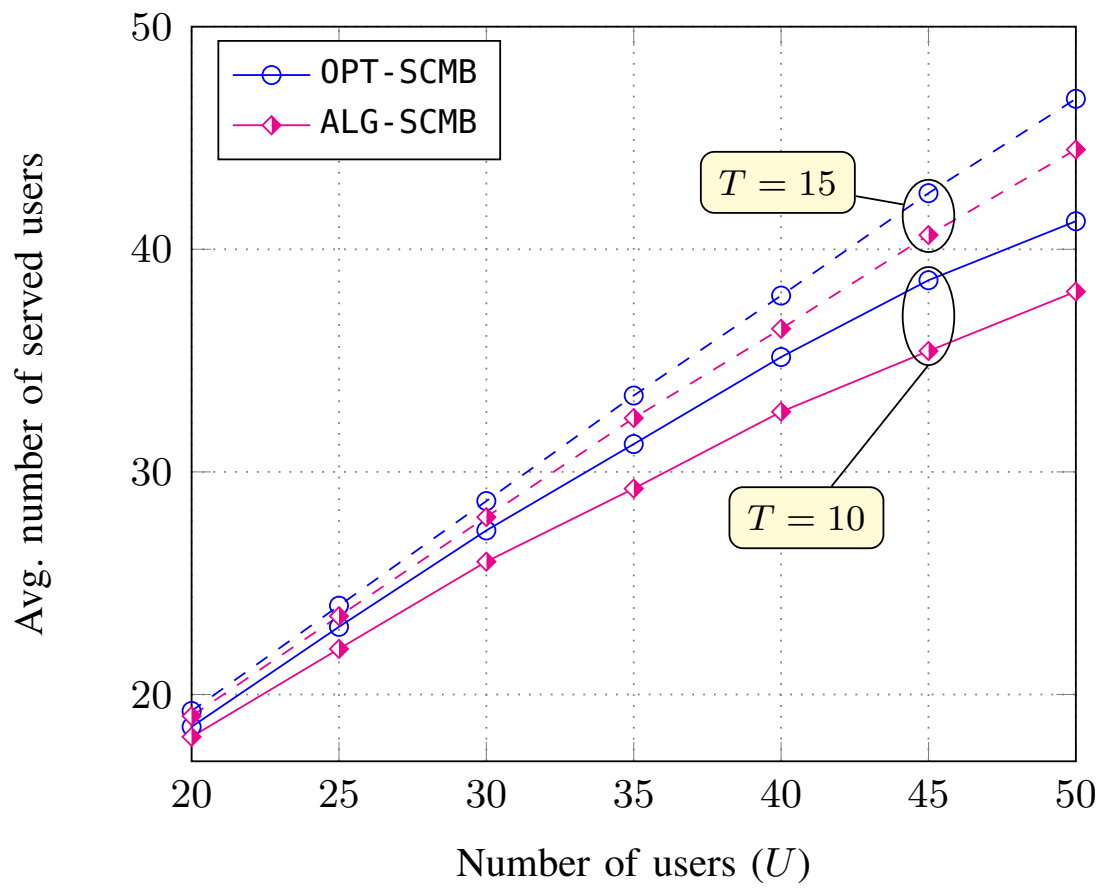

Fig. 7: Average number of served users by ALG-SCMB and OPT-SCMB for different $T$.

number of users that can be served is reached and hence the performance of the optimal algorithm saturates. We can see that as more EBSs are available, more users are served by both algorithms and, since $T$ is fixed, ALG-SCMB gets closer to OPT-SCMB. The gap between both algorithms is still small 


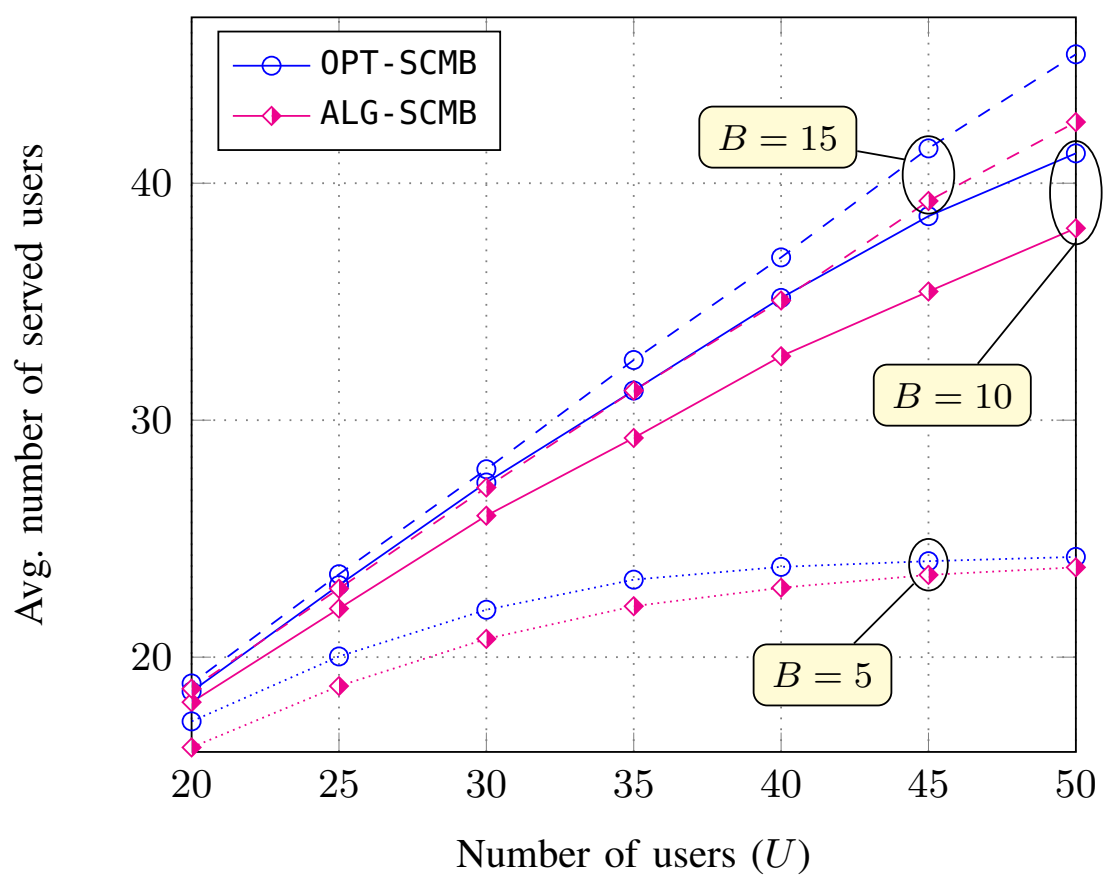

Fig. 8: Average number of served users by ALG-SCMB and OPT-SCMB for different $B$.

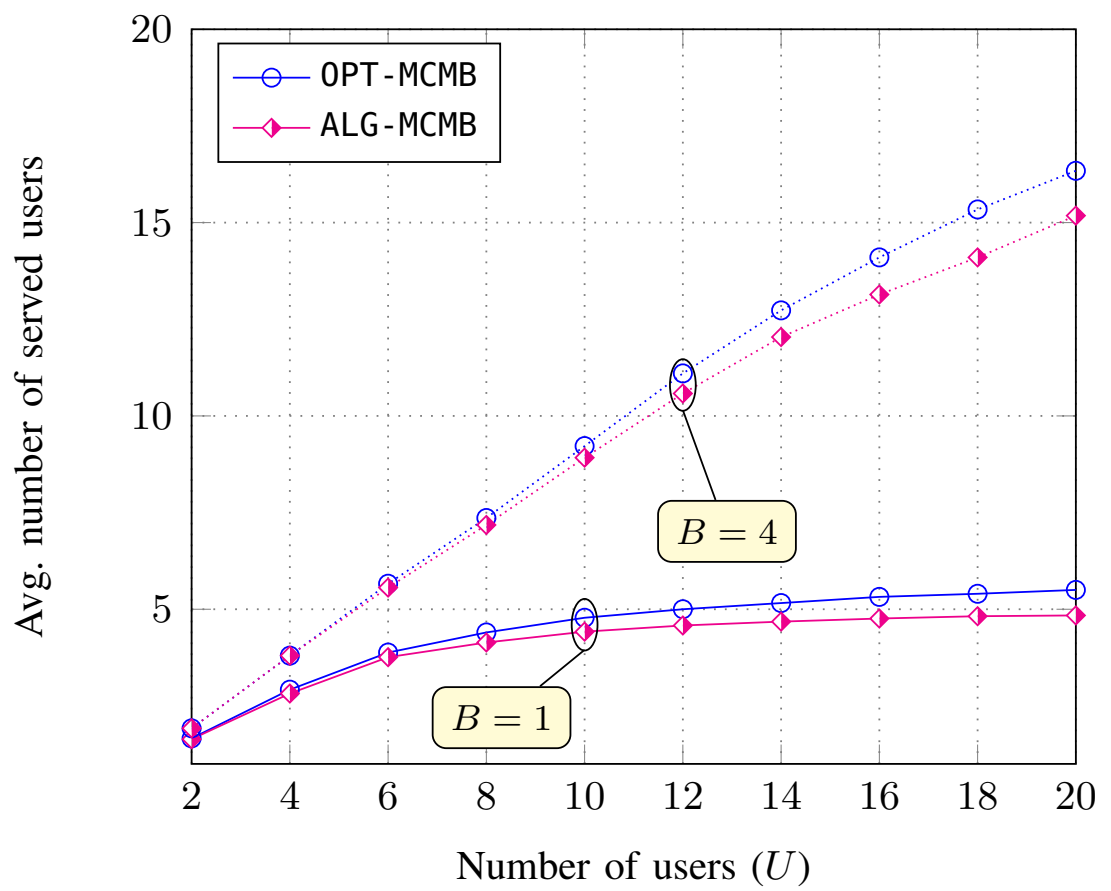

Fig. 9: Average number of served users by ALG-MCMB and OPT-MCMB for different $B(C=2)$.

even for large values of $U$ and $B$, which illustrates the superiority of ALG-SCMB.

In Fig. 9, we illustrate the performance of ALG-MCMB against OPT-MCMB. We can see that the performance of ALG-MCMB is close-to-optimal. When the number of EBSs increases, more users are 
served even when the energy is constant (i.e., for fixed $\lambda$ ). From Fig. 9, we can see that ALG-MCSB has close-to-optimal performance with a ratio (heuristic-to-optimal) of 0.88 for $U=20$ and $B=1$. This ratio becomes equal to 0.93 for $U=20$ and $B=4$. Hence, we conclude that the performance ratio increases with more EBSs in the network, which illustrates the efficiency of ALG-MCMB.

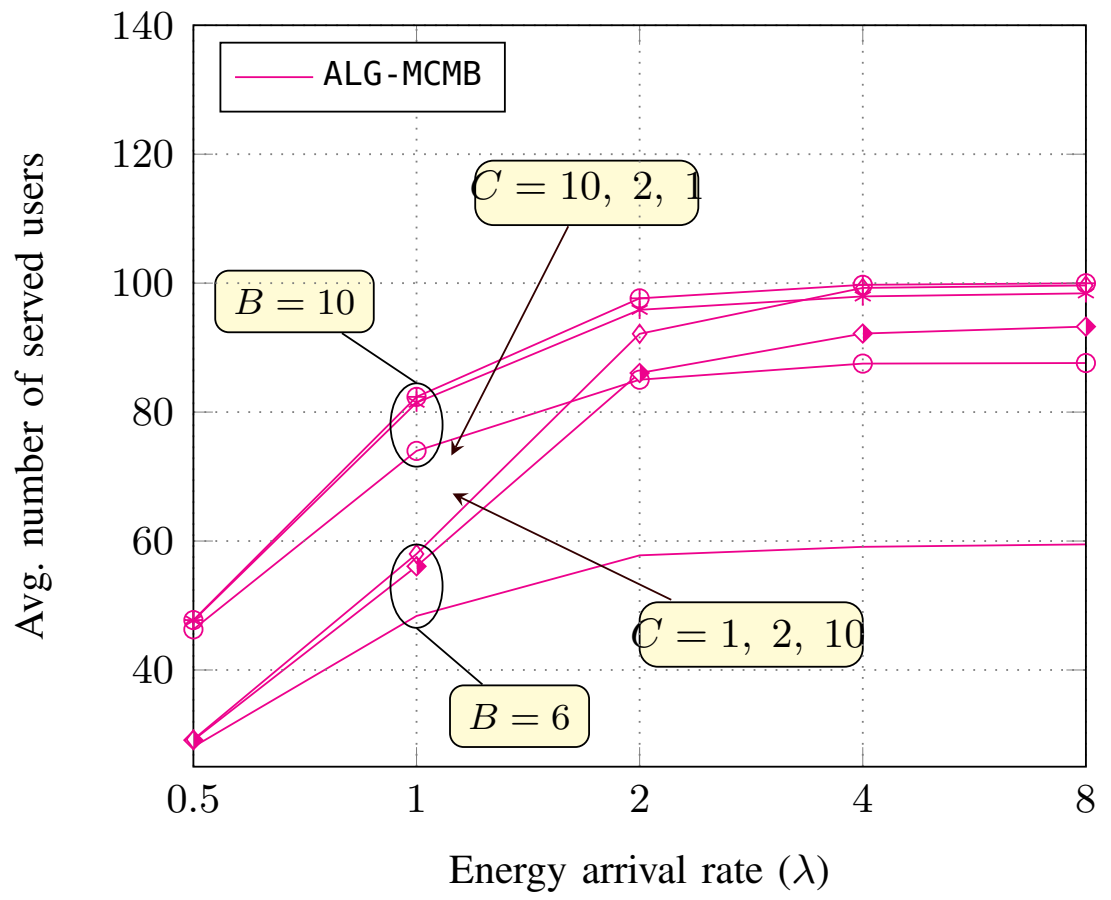

Fig. 10: Average number of served users by ALG-MCMB for different $B$ and $C(U=100)$.

Fig. 10 illustrates the performance of ALG-MCMB for large number of users, $U=100$. (The optimal algorithm cannot be obtained for computational running-time complexity issues.) We can observe that ALG-MCMB serves more users as $\lambda, B$, or $C$ increases. Importantly, the number of served users by ALG-MCMB increases faster with $B$ than with $C$. For example, on the one hand, for $\lambda=1$ and when $B$ varies from 6 to 10, ALG-MCMB serves 1.45 times more users for $C=2$ and 1.42 times more users for $C=10$. On the other hand, for $\lambda=1$ and when $C$ varies from 2 to 10, ALG-MCMB serves 1.035 times more users for $B=6$ and serves 1.011 times more users for $B=10$. We can conclude form Fig. 10 that (i) having more channels is advantageous (in terms of maximizing the number of users) when $\lambda$ is large and $B$ is fixed, and (ii) having more BSs is advantageous when $\lambda$ is small and $C$ is fixed.

\section{CONCLUSION}

In this paper, we studied a resource allocation problem in green dense cellular networks. This problem, called resource allocation with energy and deadlines constraints $(R A E D)$, involved user association, 
scheduling with hard deadlines and channel allocation where base stations (BSs) are solely powered by harvested energy. First, we modeled RAED as integer linear program. Next, we characterized the computational complexity of four cases of $R A E D$ by studying their $\mathcal{N} \mathcal{P}$-hardness. For the case of a single channel and a single BS, we proposed two optimal polynomial-time algorithms - the second being much less complex that only works under a common deadlines assumption. These two proposed algorithms were shown to have robust performance against various parameters changes. For the case of a single channel and multiple BSs, RAED was shown to be $\mathcal{N} \mathcal{P}$-hard and a $\frac{1}{2}$-approximation algorithm was designed to solve it. The average performance of this approximation algorithm was shown to be higher than the theoretical performance guarantee. Next, we studied the case of multiple channels (with both single and multiple $\mathrm{BSs}$ ). We showed that RAED is $\mathcal{N} \mathcal{P}$-hard even with a single $\mathrm{BS}$ and two channels and we designed a heuristic algorithm to solve both cases. We showed that this heuristic algorithm has close-to-optimal performance.

As for future work, we will solve RAED in the online settings with fairness constraints between users. Our objective will be to develop competitive and fair algorithms that perform well compared to a omniscient offline algorithm.

\section{REFERENCES}

[1] J. G. Andrews, S. Buzzi, W. Choi, S. V. Hanly, A. Lozano, A. C. K. Soong, and J. C. Zhang, "What Will 5G Be?” IEEE J. Sel. Areas Commun., vol. 32, no. 6, pp. 1065-1082, Jun. 2014.

[2] A. Damnjanovic, J. Montojo, Y. Wei, T. Ji, T. Luo, M. Vajapeyam, T. Yoo, O. Song, and D. Malladi, "A Survey On 3GPP Heterogeneous Networks," IEEE Wireless Commun., vol. 18, no. 3, pp. 10-21, Jun. 2011.

[3] G. Liu, X. Hou, J. Jin, F. Wang, Q. Wang, Y. Hao, Y. Huang, X. Wang, X. Xiao, and A. Deng, "3-D-MIMO With Massive Antennas Paves the Way to 5G Enhanced Mobile Broadband: From System Design to Field Trials," IEEE J. Sel. Areas Commun., vol. 35, no. 6, pp. 1222-1233, Jun. 2017.

[4] H. Elsawy, E. Hossain, and D. I. Kim, "HetNets with Cognitive Small Cells: User Offloading and Distributed Channel Access Techniques," IEEE Commun. Mag., vol. 51, no. 6, pp. 28-36, Jun. 2013.

[5] B. Zhuang, D. Guo, E. Wei, and M. L. Honig, "Scalable Spectrum Allocation and User Association in Networks With Many Small Cells," IEEE Trans. Commun., vol. 65, no. 7, pp. 2931-2942, Jul. 2017.

[6] B. Wang, Q. Kong, W. Liu, and L. T. Yang, “On Efficient Utilization of Green Energy in Heterogeneous Cellular Networks,” IEEE Systems Journal, vol. 11, no. 2, pp. 846-857, Jun. 2017.

[7] B. Zhuang, D. Guo, E. Wei, and M. L. Honig, "Large-Scale Spectrum Allocation for Cellular Networks via Sparse Optimization," IEEE Trans. Signal Process., vol. 66, no. 20, pp. 5470-5483, Oct. 2018.

[8] B. Zhuang, D. Guo, and M. L. Honig, "Energy-Efficient Cell Activation, User Association, and Spectrum Allocation in Heterogeneous Networks," IEEE J. Sel. Areas Commun., vol. 34, no. 4, pp. 823-831, Apr. 2016.

[9] J. Zhao, Y. Liu, K. K. Chai, A. Nallanathan, Y. Chen, and Z. Han, "Spectrum Allocation and Power Control for Non-Orthogonal Multiple Access in HetNets," IEEE Trans. Wireless Commun., vol. 16, no. 9, pp. 5825-5837, Sept. 2017.

[10] Y. Lin, W. Bao, W. Yu, and B. Liang, "Optimizing User Association and Spectrum Allocation in HetNets: A Utility Perspective," IEEE J. Sel. Areas Commun., vol. 33, no. 6, pp. 1025-1039, Jun. 2015. 
[11] C. Huang, J. Zhang, H. V. Poor, and S. Cui, "Delay-Energy Tradeoff in Multicast Scheduling for Green Cellular Systems," IEEE J. Sel. Areas Commun., vol. 34, no. 5, pp. 1235-1249, May 2016.

[12] A. Deshmukh and R. Vaze, "Online Energy-Efficient Packet Scheduling for a Common Deadline With and Without Energy Harvesting," IEEE J. Sel. Areas Commun., vol. 34, no. 12, pp. 3661-3674, Dec. 2016.

[13] S. Krishnasamy and S. Shakkottai, "Spectrum Sharing and Scheduling in D2D-enabled Dense Cellular Networks," in Proc. Int. Symp. on Modeling and Optim. in Mobile, Ad Hoc, and Wireless Netw. (WiOpt), May 2015, pp. 307-314.

[14] Y. C. Wang and S. T. Chen, "Delay-Aware ABS Adjustment to Support QoS for Real-Time Traffic in LTE-A HetNet," IEEE Wireless Commun. Lett., vol. 6, no. 5, pp. 590-593, Oct. 2017.

[15] V. Sciancalepore, I. Filippini, V. Mancuso, A. Capone, and A. Banchs, "A Semi-Distributed Mechanism for Inter-Cell Interference Coordination Exploiting the ABSF Paradigm,” in Proc. IEEE Int. Conf. on Sensing, Commun., and Networking (SECON), Jun. 2015, pp. 193-201.

[16] Z. Mlika, E. Driouch, and W. Ajib, "User Association and Scheduling With Hard Deadlines in Heterogeneous Cellular Networks," IEEE Commun. Lett., vol. 21, no. 12, pp. 2698-2701, Dec. 2017.

[17] M. Chetto, "Optimal Scheduling for Real-Time Jobs in Energy Harvesting Computing Systems," IEEE Trans. Emerg. Topics Comput., vol. 2, no. 2, pp. 122-133, Jun. 2014.

[18] H. Wang, J. X. Zhang, and F. Li, "Worst-case Performance Guarantees of Scheduling Algorithms Maximizing Weighted Throughput in Energy Harvesting Networks," Sustainable Computing: Informatics and Systems, vol. 4, no. 3, pp. 172 - $182,2014$.

[19] F. Shan, J. Luo, W. Wu, M. Li, and X. Shen, "Discrete Rate Scheduling for Packets With Individual Deadlines in Energy Harvesting Systems," IEEE J. Sel. Areas Commun., vol. 33, no. 3, pp. 438-451, Mar. 2015.

[20] A. Hentati, J. F. Frigon, , and W. Ajib, "Information Age and Packet Loss Performance Analysis of Energy Harvesting WSNs," in Proc. IEEE Vehicular Technology Conference (VTC-Fall), Sept. 2018, pp. 1-5.

[21] IBM, “IBM ILOG CPLEX Optimizer," 2010. [Online]. Available: http://www-01.ibm.com/software/integration/optimization/ cplex-optimizer/

[22] A. Schrijver, Theory of Linear and Integer Programming. New York, NY, USA: John Wiley \& Sons, Inc., 1986.

[23] C. Chekuri and S. Khanna, "A Polynomial Time Approximation Scheme for the Multiple Knapsack Problem," SIAM Journal on Computing, vol. 35, no. 3, pp. 713-728, 2005.

[24] M. R. Garey and D. S. Johnson, Computers and Intractability: A Guide to the Theory of NP-Completeness. New York, NY, USA: W. H. Freeman \& Co., 1979.

[25] M. L. Pinedo, Scheduling: Theory, Algorithms, and Systems, 5th ed. Springer Publishing Company, Incorporated, 2016.

[26] D. P. Williamson and D. B. Shmoys, The Design of Approximation Algorithms, 1st ed. New York, NY, USA: Cambridge University Press, 2011.

[27] 3GPP, "Further Advancements for E-UTRA Physical Layer Aspects (Release 9)," Tech. Rep. 3GPP TR 36.814, Mar. 2010, v9.0.0.

[28] S. Mitchell, M. O’Sullivan, and I. Dunning, "PuLP: A Linear Programming Toolkit for Python," The University of Auckland, Department of Engineering Science, Tech. Rep., Sept. 2011. 